\title{
Sub-millitesla magnetic field effects on the recombination reaction of flavin and ascorbic acid radicals
}

Emrys W. Evans, Daniel R. Kattnig, Kevin B. Henbest, P. J. Hore, Stuart R. Mackenzie, and Christiane R. Timmel

Citation: The Journal of Chemical Physics 145, 085101 (2016); doi: 10.1063/1.4961266

View online: https://doi.org/10.1063/1.4961266

View Table of Contents: http://aip.scitation.org/toc/jcp/145/8

Published by the American Institute of Physics

\section{Articles you may be interested in}

Spin relaxation of radicals in cryptochrome and its role in avian magnetoreception

The Journal of Chemical Physics 145, 035104 (2016); 10.1063/1.4958624

Floquet theory of radical pairs in radiofrequency magnetic fields

The Journal of Chemical Physics 145, 124117 (2016); 10.1063/1.4963793

An efficient quantum mechanical method for radical pair recombination reactions

The Journal of Chemical Physics 145, 244101 (2016); 10.1063/1.4972277

Magnetoelectroluminescence in organic light-emitting diodes

The Journal of Chemical Physics 144, 214109 (2016); 10.1063/1.4953093

An improved semiclassical theory of radical pair recombination reactions

The Journal of Chemical Physics 139, 124106 (2013); 10.1063/1.4821817

Photofragmentation dynamics and dissociation energies of $\mathrm{MoO}$ and $\mathrm{CrO}$

The Journal of Chemical Physics 147, 013921 (2017); 10.1063/1.4979979

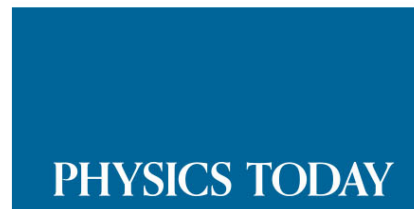




\title{
Sub-millitesla magnetic field effects on the recombination reaction of flavin and ascorbic acid radicals
}

\author{
Emrys W. Evans, ${ }^{1, a)}$ Daniel R. Kattnig, ${ }^{2, a)}$ Kevin B. Henbest, ${ }^{1}$ P. J. Hore, ${ }^{2}$ \\ Stuart R. Mackenzie, ${ }^{2, b)}$ and Christiane R. Timmel ${ }^{1, b)}$ \\ ${ }^{1}$ Department of Chemistry, Centre for Advanced Electron Spin Resonance, University of Oxford, Oxford, \\ United Kingdom \\ ${ }^{2}$ Physical and Theoretical Chemistry Laboratory, Department of Chemistry, University of Oxford, Oxford, \\ United Kingdom
}

(Received 8 July 2016; accepted 5 August 2016; published online 24 August 2016)

\begin{abstract}
Even though the interaction of a $<1 \mathrm{mT}$ magnetic field with an electron spin is less than a millionth of the thermal energy at room temperature $\left(k_{\mathrm{B}} T\right)$, it still can have a profound effect on the quantum yields of radical pair reactions. We present a study of the effects of sub-millitesla magnetic fields on the photoreaction of flavin mononucleotide with ascorbic acid. Direct control of the reaction pathway is achieved by varying the rate of electron transfer from ascorbic acid to the photo-excited flavin. At pH 7.0, we verify the theoretical prediction that, apart from a sign change, the form of the magnetic field effect is independent of the initial spin configuration of the radical pair. The data agree well with model calculations based on a Green's function approach that allows multinuclear spin systems to be treated including the diffusive motion of the radicals, their spin-selective recombination reactions, and the effects of the inter-radical exchange interaction. The protonation states of the radicals are uniquely determined from the form of the magnetic field-dependence. At $\mathrm{pH} 3.0$, the effects of two chemically distinct radical pair complexes combine to produce a pronounced response to $\sim 500 \mu \mathrm{T}$ magnetic fields. These findings are relevant to the magnetic responses of cryptochromes (flavin-containing proteins proposed as magnetoreceptors in birds) and may aid the evaluation of effects of weak magnetic fields on other biologically relevant electron transfer processes. Published by AIP Publishing. [http://dx.doi.org/10.1063/1.4961266]
\end{abstract}

\section{INTRODUCTION}

The effects of applied magnetic fields on photochemical reactions that have free radicals as intermediates can be explained by the radical pair mechanism. ${ }^{1,2}$ The key elements of the mechanism are the photo-induced formation of an electron-spin-correlated radical pair, its coherent spin dynamics driven by hyperfine, Zeeman, and other interactions, and spin-selective radical recombination reactions. An intriguing aspect of the mechanism is that it satisfactorily accounts for the sensitivity of chemical reactions to magnetic interactions that are as small as $10^{-6} k_{\mathrm{B}} T$ under conditions where equilibrium effects would be entirely negligible. In this context, photochemical radical pair reactions in the retinas of migratory birds are thought to be responsible for the birds' ability to sense the direction of the Earth's magnetic field (25-65 $\mu \mathrm{T}$ ) for the purpose of navigation. ${ }^{3}$ There have even been suggestions that the radical pair mechanism might be relevant to the purported biological effects of extremely low frequency (e.g., 50/60 Hz) anthropogenic electromagnetic fields. ${ }^{4}$

A feature of the radical pair mechanism relevant for magnetic fields weaker than a few millitesla is the socalled low field effect (LFE). ${ }^{5,6}$ While LFEs in a $\sim 50 \mu \mathrm{T}$

\footnotetext{
a)E. W. Evans and D. R. Kattnig contributed equally to this work.

b) Authors to whom correspondence should be addressed. Electronic addresses: christiane.timmel@chem.ox.ac.uk and stuart.mackenzie@chem. ox.ac.uk
}

magnetic field have so far only been demonstrated for a carefully chosen molecular triad at low temperature, ${ }^{7}$ $\sim 1 \mathrm{mT}$ magnetic field effects (MFEs) are well-documented, in particular for exciplex-forming donor-acceptor systems in low-polarity organic solvents. ${ }^{8,9}$ LFEs have also been seen in the conductance and electroluminescence of organic diodes, where they are known as "ultra-small magnetic field effects." 10,11 Only a few reaction systems are known to exhibit LFEs in aqueous solution. A small effect has been detected in an in vitro study of a cryptochrome protein (the putative magnetic compass sensor in birds) and a closely related DNA-photolyase. ${ }^{12}$ For the former, the LFE peaks at $1.5 \mathrm{mT}$ and amounts to about $10 \%$ of the limiting effect at high field. In both proteins, the radical pair comprises the semi-reduced form of the flavin adenine dinucleotide (FAD) co-factor and a semi-oxidized tryptophan residue. A very recent study has demonstrated a LFE for the intramolecular radical pair formed by photolysis of FAD in solution. ${ }^{13}$ With an amplitude of only $3.4 \%$ relative to the effect at high field, its detection required the development of sensitive spectroscopic techniques.

Although bimolecular donor-acceptor systems are expected to give rise to LFEs, such effects in aqueous solution are usually harder to observe than for their intramolecular counterparts, mainly because of the low probability of radical re-encounters. As a consequence, and despite extensive efforts, LFEs of intermolecular radical pairs in aqueous solution have remained a rarity. In a recent study, ${ }^{14}$ a prominent LFE was 
observed for the photo-reaction of flavin mononucleotide (FMN) with ascorbic acid $\left(\mathrm{AscH}_{2}\right)$ in aqueous solution. Under conditions of continuous illumination, the magnetic field effects were found to be amplified by slow radicaltermination reactions, the amplification being larger in the low field region than at higher fields. Ascorbic acid was recently suggested as a possible radical pair partner for the FAD radical in a cryptochrome magnetoreceptor. ${ }^{15}$ The small hyperfine interactions in the ascorbyl radical could result in a more sensitive magnetic compass than the originally proposed radical pair containing a tryptophan radical. ${ }^{16}$

In this report, we present a detailed study of LFEs on the photoreaction of FMN with ascorbic acid. Given the widespread biological occurrence of both flavins and ascorbic acid (vitamin C), we hope that our findings may be of help in evaluating the effects of weak magnetic fields on biologically relevant charge-transfer reactions. ${ }^{17,18}$

\section{PHOTOCHEMISTRY OF FMN AND ASCORBIC ACID}

Magnetic field effects on the photochemistry of FMN in the presence of ascorbic acid can broadly be understood by means of the reaction scheme in Fig. 1(a). Electron transfer from an ascorbic acid molecule to the photo-excited flavin produces geminate radical pairs either directly via the excited singlet state (S-pathway) or, after intersystem crossing, via the excited triplet state (T-pathway). This occurs with conservation of spin angular momentum so that the radical pair is initially spin-correlated, with the same spin-multiplicity as its precursor. Once the radicals have diffused far enough apart that their mutual exchange interaction is small, the hyperfine interactions in the radicals induce coherent interconversion of the singlet and triplet states. The geminate radical pair recombines exclusively by reverse electron transfer from its singlet state. The triplet radical pair cannot recombine because the excited triplet states of both FMN and ascorbic acid are energetically inaccessible. Those geminate radicals that do not recombine diffuse apart to form free radicals which encounter at a later time, having lost their spin-correlation, to form singlet and triplet radical pairs ("F-pairs") with a 1:3 statistical ratio. The efficiency of singlet-triplet interconversion is reduced by the Zeeman interaction of the electron spins with an applied magnetic field which shifts the energies of two of the three triplet states away from that of the singlet. As a result, the probability that the geminate radical pair recombines, rather than separating, depends on the strength of the magnetic field. ${ }^{1,2,19}$

A typical response of the photocycle to an applied magnetic field, known as the MARY (magnetically altered reaction yield) profile, is shown schematically in Fig. 1(b) where the fractional magnetic field effect (MFE, defined below) is plotted against the field strength. Ignoring effects arising from spin relaxation, and from the difference in the $g$-values of the two radicals (negligible for the magnetic fields considered here), the MFE saturates when the Zeeman interaction is much larger than the hyperfine interactions. Under appropriate conditions, the MFE is found to change sign (Fig. 1(b)) for weaker magnetic fields, when the Zeeman and hyperfine interactions are comparable in strength. This increase in the efficiency of singlet-triplet interconversion is the LFE and it arises because a weak field removes degeneracies amongst the zero-field spin states. ${ }^{5}$ The LFE can be observed when the radical pair lifetime is long compared to the reciprocal of the relevant energy-level splittings but not so long that spin relaxation destroys the spin-correlation before the radicals can recombine.

The structures of FMN and $\mathrm{AscH}_{2}$ are given in Fig. 1(c). a

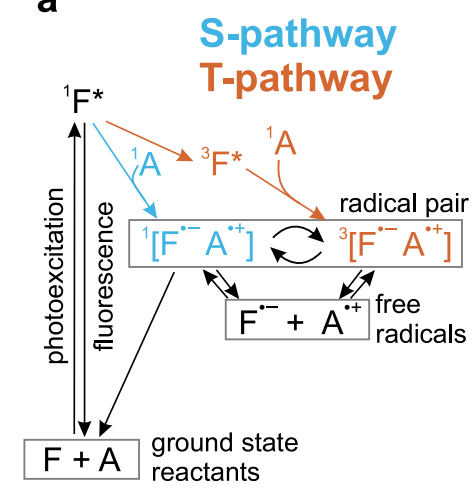

b

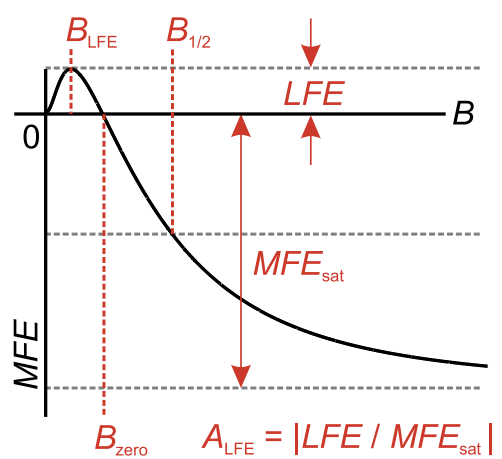

C

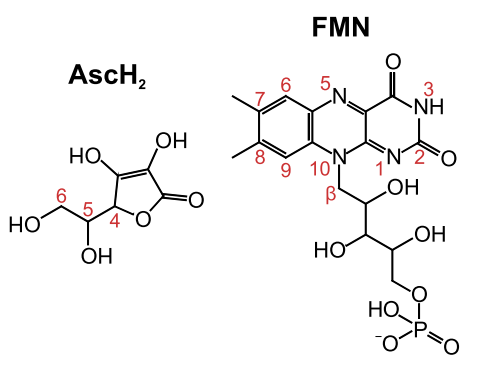

FIG. 1. (a) Photochemical reaction scheme for FMN (F) and ascorbic acid (A). Depending on the rate of intermolecular electron transfer, the radical pair [F ${ }^{\cdot-}$ $\mathrm{A}^{\cdot+}$ ] is formed either directly from the FMN excited singlet state (S-pathway, blue) or, following intersystem crossing, via the triplet excited state (T-pathway, orange). Conservation of spin angular momentum means that the $\mathrm{S}$ - and T-pathways produce radical pairs in singlet, ${ }^{1}\left[\mathrm{~F}^{\cdot-} \mathrm{A}^{\cdot+}\right]$, and triplet, ${ }^{3}\left[\mathrm{~F}^{\cdot-} \mathrm{A}^{\cdot+}\right]$, states, respectively. The interconversion of ${ }^{1}\left[\mathrm{~F}^{\cdot-} \mathrm{A}^{\cdot+}\right]$ and ${ }^{3}\left[\mathrm{~F}^{--} \mathrm{A}^{++}\right]$is driven by hyperfine interactions and modulated by the electron Zeeman interaction with an external magnetic field. Because the radical pair recombines exclusively from its singlet state, and since the ground state $(\mathrm{F}+\mathrm{A})$ and the free radicals $\left(\mathrm{F}^{\cdot-}+\mathrm{A}^{\cdot+}\right)$ are the only long-lived $(\geq 1 \mu \mathrm{s})$ species, the Zeeman interaction manifests itself as magnetic field effects on the photostationary populations of $\mathrm{F}+\mathrm{A}$ and $\mathrm{F}^{\cdot-}+\mathrm{A}^{\cdot+}$ under conditions of continuous illumination. The protonation states of the various $\mathrm{F}$ and $\mathrm{A}$ species are not shown. The slow reaction steps discussed in Ref. $14\left(\mathrm{~F}^{\bullet^{-}} \rightarrow \mathrm{F}_{\text {and }} \mathrm{A}^{++} \rightarrow \mathrm{A}\right)$ are irrelevant for the experiments described here and have not been included in the reaction scheme. (b) Schematic MARY profile showing the dependence of the magnetic field effect (MFE, Eq. (1)) on the strength of the applied magnetic field. The definitions of the four MARY parameters are indicated. This profile is appropriate for the singlet recombination product of a radical pair initially formed in a triplet state. (c) Chemical structures of flavin mononucleotide and ascorbic acid. 


\section{EXPERIMENTAL METHODS}

Radical pairs were formed by continuous photoexcitation of flowing $\left(0.5 \mathrm{~mL} \mathrm{~min}{ }^{-1}\right)$ aqueous $\mathrm{FMN}+\mathrm{AscH}_{2}$ solutions using a $405 \mathrm{~nm}$ diode laser (Power Technology, $350 \mathrm{~mW}$ ). As described in Refs. 14 and 20, MFEs were detected by monitoring the fluorescence of the excited singlet state of FMN $\left({ }^{1} \mathrm{~F}^{*} \rightarrow \mathrm{F}\right.$ in Fig. 1(a)) in the wavelength range $500-600 \mathrm{~nm}$. The magnetic field effect is reported as

$$
\operatorname{MFE}(B)=\frac{I_{\mathrm{F}}(B)-I_{\mathrm{F}}(0)}{I_{\mathrm{F}}(0)},
$$

where $I_{\mathrm{F}}(B)$ is the fluorescence intensity in the photostationary state in the presence of an applied magnetic field of strength $B . I_{\mathrm{F}}(B)$ was measured with $0.10 \mathrm{mT}$ and $1.15 \mathrm{mT}$ field increments for $B \leq 2.70 \mathrm{mT}$ and $B>2.70 \mathrm{mT}$, respectively. All measurements were performed at $21^{\circ} \mathrm{C}$. Further experimental details can be found in Ref. 20.

The FMN concentration was $10 \mu \mathrm{M}$ in all MFE measurements. The ascorbic acid concentration was in the range 2-300 mM. Samples were prepared in formate buffer $(\mathrm{pH} 3.0$, ionic strength $=0.1 \mathrm{M})$ or in phosphate buffer $(\mathrm{pH}$ 7.0 , ionic strength $=0.1 \mathrm{M}$, except for the highest $\left[\mathrm{AscH}_{2}\right]$, where the ionic strength was determined by the ascorbic acid) using ultrapure Millipore water (resistivity, 18.2 M $\Omega \mathrm{cm}$ ). Chemicals were purchased from Sigma Aldrich and used without further purification.

A Varian Cary Eclipse spectrometer was employed for the fluorescence quenching measurements at an FMN concentration of $1 \mu \mathrm{M}$ at $21^{\circ} \mathrm{C}$.

EPR spectra were recorded using a Bruker Elexsys 680 spectrometer with a high-sensitivity ER 4119 HS-W1 resonator at $21^{\circ} \mathrm{C}$. Samples of the $\mathrm{FMNH}^{\circ}$ radical were prepared at $\mathrm{pH} 7.0$ (phosphate buffer) under an atmosphere of nitrogen in a glove box by comproportionation of a 1:1 mixture (2.6 mM) of FMN and its fully reduced form, $\mathrm{FMNH}_{2}$. The latter was obtained by passing a solution of FMN through a Jones reductor (zinc amalgam prepared by reacting granular $\mathrm{Zn}$ metal with a $2 \% \mathrm{v} / \mathrm{v}$ aqueous solution of $\mathrm{HgCl}_{2}$ ).

Transient absorption data were recorded at $21^{\circ} \mathrm{C}$ using a home-built apparatus. Samples were held in a quartz cuvette (Hellma 104.002F QS, $10 \mathrm{~mm}$ path-length, internal dimensions $2 \times 10 \times 45 \mathrm{~mm}$ ) and excited using a dye laser (Sirah Cobra) pumped by a Nd:YAG laser (Continuum Surelite 1). The output of the dye laser was $\sim 5 \mathrm{~mJ}$ per pulse at $450 \mathrm{~nm}$ with a repetition rate of $1 \mathrm{~Hz}$. A $1000 \mathrm{~W}$ xenon arc lamp supplied the probe light in an orthogonal direction. A water filter and a long-pass filter were used to remove infrared and ultraviolet components in order to protect the samples. Transient absorption data at specified wavelengths were recorded using a monochromator (Oriel 77250) and a photomultiplier tube (Hamamatsu R928) coupled to an oscilloscope (LeCroy Waverunner LT342L). Further details can be found in Refs. 12 and 21.

Isotropic hyperfine coupling constants were calculated using density functional theory (DFT) in Gaussian 09, Revision D.01. ${ }^{22}$ The following protocols were used: B3LYP/6$311+\mathrm{G}(\mathrm{d}, \mathrm{p}) / / \mathrm{B} 3 \mathrm{LYP} / \mathrm{EPR}-\mathrm{III}$ for the ascorbyl radicals $\left(\mathrm{Asc}^{--}\right.$, AscH ${ }^{\cdot}$ and $\mathrm{AscH}_{2}{ }^{++}$) and B3LYP/6-311G(d,p)//B3LYP/EPRIII for the FMN radicals $\left(\mathrm{FMNH}^{-}\right.$and $\mathrm{FMN}^{\cdot-}$ ). In the final single-point calculation, the cc-pVTZ basis set was used for the phosphorus atom in the flavin radicals. In all cases, the water environment was modelled using the Integral Equation Formalism of the Polarizable Continuum Model (IEFPCM).

\section{EXPERIMENTAL RESULTS}

The photochemistry of FMN in the presence of $\mathrm{AscH}_{2}$ was summarized above and shown in Fig. 1(a). In the absence of electron donors, the excited singlet state of FMN has a reported ${ }^{23}$ lifetime of $4.6 \mathrm{~ns}$ and displays a bright green fluorescence with a quantum yield of 0.27 , which, under the conditions of continuous photoexcitation employed here, serves as a sensitive indicator of the ground state concentration. As demonstrated in Ref. 20, the photostationary concentration of the ground state of the flavin, and therefore the fluorescence intensity, can be affected by applied magnetic fields via changes in the branching ratio of the recombination and diffusive separation of the radicals that comprise the geminate pair.

\section{A. EPR experiments and DFT calculations}

Quantitative interpretation of the magnetic field effects on any radical pair reaction requires knowledge of the hyperfine interactions in the radicals. Those of the ascorbyl anion radical $\left(\mathrm{Asc}^{-}\right.$) have previously been measured ${ }^{24}$ those of the neutral and cation ascorbic acid radicals, $\mathrm{AscH}{ }^{-}$and $\mathrm{AscH}_{2}{ }^{+}$, and the FMN radicals, $\mathrm{FMN}^{--}$and $\mathrm{FMNH}^{-}$, have not. Section $\mathrm{S} 1$ of the supplementary material lists the isotropic hyperfine coupling constants calculated using Gaussian 09.22

The EPR spectra of FMNH in $\mathrm{H}_{2} \mathrm{O}$ and in $\mathrm{D}_{2} \mathrm{O}$ (where the acidic protons at positions 3 and 5 are substituted for deuterons) were measured as a check on the DFT values (Section S2 of the supplementary material). The isotropic hyperfine coupling constants obtained by spectral fitting generally agree with the DFT results. The hyperfine interactions used in the simulations described below are listed in Section S1 of the supplementary material.

\section{B. Transient absorption measurements}

The photochemistry was first characterised by transient absorption measurements at $\mathrm{pH} 3.0$ and $\mathrm{pH} 7.0$ with $\sim 1 \mu$ s time resolution. At low concentrations of $\mathrm{AscH}_{2}$ (e.g., $0.5 \mathrm{mM}$ ) at $\mathrm{pH} 3.0$, the protonated triplet state ${ }^{3} \mathrm{FMNH}^{+*}$ reacts to form the neutral FMNH' radical. FMNH' is also formed at $\mathrm{pH}$ 7.0, even though the transient FMN triplet $\left(\mathrm{p} K_{\mathrm{a}}=4.5^{25,26}\right)$ seems not to be protonated at this $\mathrm{pH}$. A target analysis (Section S3 of the supplementary material) indicates that this is a two-stage process in which ${ }^{3} \mathrm{FMN}^{*}$ is initially reduced to the anion radical, $\mathrm{FMN}^{--}$, which then protonates on a $\sim 1 \mu$ s timescale. These findings are consistent with Heelis et al. ${ }^{27}$

\section{Fluorescence quenching experiments}

Estimates of the proportions of geminate radical pairs formed in singlet and triplet states were obtained from steady state fluorescence quenching experiments in the presence of 
up to $25 \mathrm{mM}$ ascorbic acid. The Stern-Volmer constants were determined to be $14 \mathrm{M}^{-1}$ and $31 \mathrm{M}^{-1}$ at $\mathrm{pH} 3.0$ and $\mathrm{pH}$ 7.0, respectively. Assuming a $\mathrm{pH}$-independent lifetime of $4.6 \mathrm{~ns}$ for the excited singlet state of $\mathrm{FMN}^{23}$ and an intersystem crossing rate constant of $1.3 \times 10^{8} \mathrm{~s}^{-1}$, the probability that the radical pair is initially singlet when $\left[\mathrm{AscH}_{2}\right]=2 \mathrm{mM}$ is 0.05 at $\mathrm{pH} 3.0$ and 0.11 at $\mathrm{pH} 7.0$. When $\left[\mathrm{AscH}_{2}\right]=300 \mathrm{mM}$, these figures change to 0.88 and 0.94 , respectively (Section S4 of the supplementary material). Thus, the initial spin configuration of the radical pair can be switched from almost completely singlet to almost completely triplet by increasing the ascorbic acid concentration.

\section{Magnetic field effect measurements}

Figure 2(a) shows MARY profiles for FMN + ascorbic acid at $\mathrm{pH} 3.0$ and $\mathrm{pH} 7.0$ at an $\mathrm{AscH}_{2}$ concentration $(2 \mathrm{mM})$ low enough that the majority of the radical pairs are formed in the triplet state (T-pathway in Fig. 1(a)). The effect of a magnetic field stronger than the hyperfine interactions (e.g., $12 \mathrm{mT}$ ) is to reduce the efficiency of triplet $\rightarrow$ singlet conversion, thereby increasing the probability that the radicals separate rather than recombining from the singlet state. Under the continuous irradiation conditions of this measurement, this leads to an increase in the concentration of long-lived
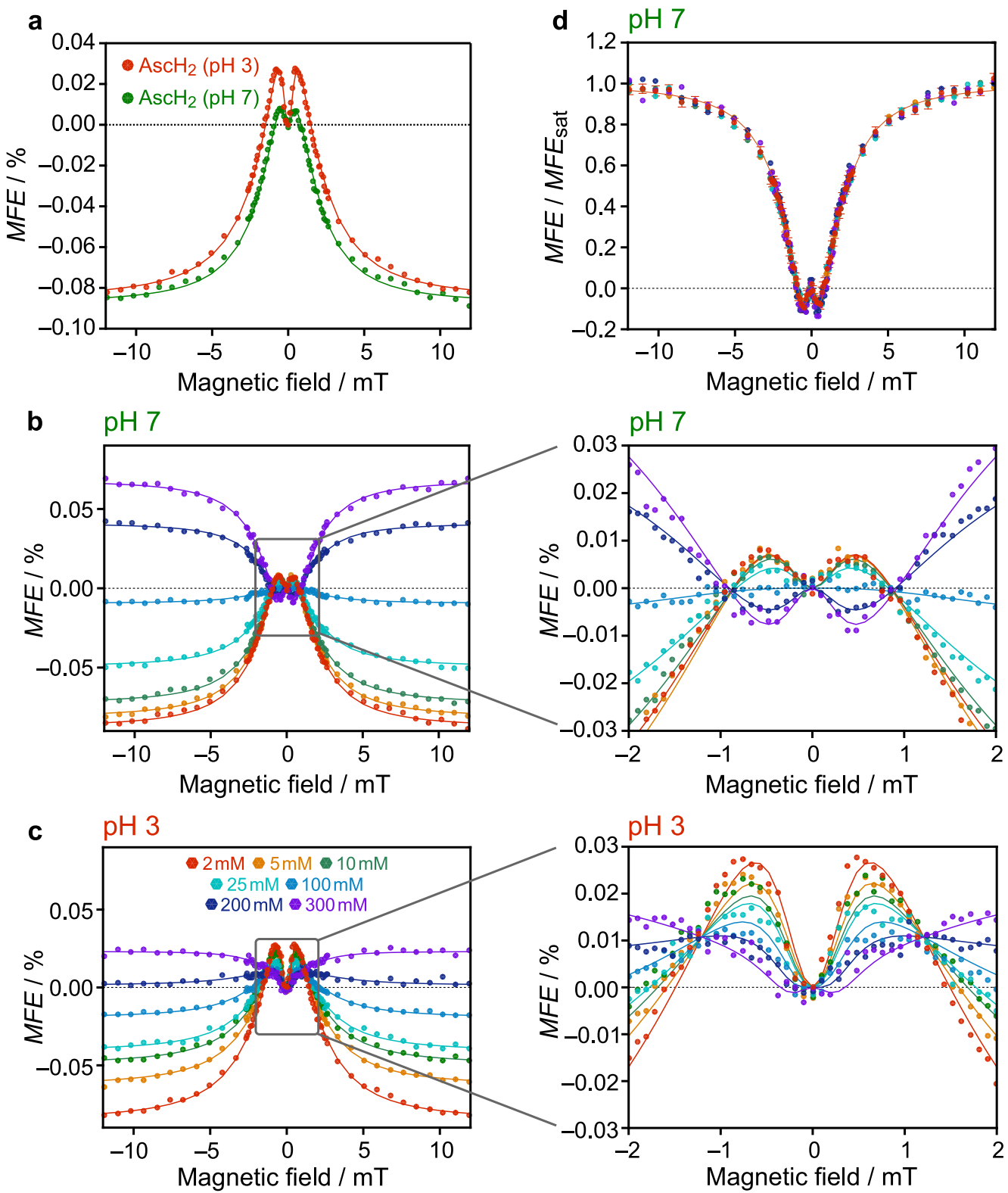

FIG. 2. (a) MARY profiles for $10 \mu \mathrm{M} F M N+2 \mathrm{mM} \mathrm{AscH}_{2}$ at $\mathrm{pH} 3.0$ (red) and $\mathrm{pH} 7.0$ (green). Negative values of $B$ correspond to inverted directions of the applied magnetic field. (b) MARY profiles for FMN $(10 \mu \mathrm{M})+\mathrm{AscH}_{2}$ at concentrations in the range 2-300 $\mathrm{mM}$ at $\mathrm{pH} 7.0$ (with an expanded view to the right). (c) MARY profiles for FMN $(10 \mu \mathrm{M})+\mathrm{AscH}_{2}$ at concentrations in the range $2-300 \mathrm{mM}$ at $\mathrm{pH} 3.0$ (with an expanded view to the right). The colour code also applies to panels (b) and (d). (d) MARY profiles from (b) scaled to $M F E_{\text {sat }}=+1$. Confidence intervals ( $99 \%$ ) are shown for the 2 mM dataset; intervals for the other scaled profiles have similar magnitudes. In all six panels the dots are the experimental measurements and the solid lines are fits to the composite lineshape model (Section S6 of the supplementary material). At both $\mathrm{pH} 3.0$ and $\mathrm{pH} 7.0$, the ionic strengths of the $\left[\right.$ Asc $\left.\mathrm{H}_{2}\right] \leq 25 \mathrm{mM}$ solutions were adjusted to $0.1 \mathrm{M}^{2}$ using appropriate concentrations of sodium chloride and sodium ascorbate. For the more concentrated solutions, the pH was adjusted through the addition of $\mathrm{NaOH}$ and $\mathrm{HCl}$. 
free radicals, a corresponding depletion of the ground state, and consequently a reduction in the fluorescence intensity. The limiting magnetic field effect at high field, $M F E_{\mathrm{sat}}$, is therefore negative (Eq. (1)). At lower fields $(\sim 1 \mathrm{mT})$, a marked LFE is observed at both $\mathrm{pH}$ values. At $\mathrm{pH} 3.0$, its amplitude is $\sim 30 \%$ of $M F E_{\text {sat }}$ when $\left[\mathrm{AscH}_{2}\right]=2 \mathrm{mM}$ and $\sim 40 \%$ when $\left[\mathrm{AscH}_{2}\right]=25 \mathrm{mM}$. At $\mathrm{pH} 7.0$, the LFE is $\sim 9 \%$ of $M F E_{\text {sat }}$, essentially independent of $\mathrm{AscH}_{2}$ concentration (see also Fig. 2(d)). The maximum of the LFE occurs at $\sim 0.6 \mathrm{mT}$ at $\mathrm{pH} 3.0$ and $\sim 0.45 \mathrm{mT}$ at $\mathrm{pH} 7.0$. These LFEs are larger (relative to $M F E_{\mathrm{sat}}$ ) and narrower than any previously observed for flavin photo-reactions. ${ }^{12,13}$

The dependence of the MARY profile on the concentration of $\mathrm{AscH}_{2}$ at $\mathrm{pH} 7.0$ and $\mathrm{pH} 3.0$ is shown in Figs. 2(b) and 2(c), respectively. In both cases, increasing $\left[\mathrm{AscH}_{2}\right]$ from $2 \mathrm{mM}$ to $300 \mathrm{mM}$ changes the sign of $M F E_{\mathrm{sat}}$ from negative to positive, consistent with the expected switch in the initial state of the radical pair from predominantly triplet to predominantly singlet. At $\mathrm{pH} 7.0$, high $\mathrm{AscH}_{2}$ concentrations also invert the sign of the LFE. At $\mathrm{pH}$ 3.0, however, the LFE is no longer visible when $\left[\mathrm{AscH}_{2}\right]=300 \mathrm{mM}$. Additional measurements at $\mathrm{pH} 4$ resemble those at $\mathrm{pH} 3.0$ (Section S5 of the supplementary material).

To facilitate a systematic evaluation of the LFE and the shape of the MARY profiles, we define four characteristic parameters (Fig. 1(b)): (i) $B_{1 / 2}$, the magnetic field at which the MFE is equal to half its limiting value, i.e., $\operatorname{MFE}\left(B_{1 / 2}\right)=0.5 M F E_{\mathrm{sat}}$; (ii) $B_{\mathrm{LFE}}$, the magnetic field at which the LFE has its maximum amplitude; (iii) $A_{\mathrm{LFE}}$, the amplitude of the LFE relative to the limiting MFE, i.e., $A_{\mathrm{LFE}}=\left|M F E\left(B_{\mathrm{LFE}}\right) / M F E_{\mathrm{sat}}\right|$; and (iv) $B_{\text {zero }}$, the magnetic field at which the MFE passes through zero going from the low-field to the high-field region, i.e., $\operatorname{MFE}\left(B_{\text {zero }}\right)=0$. Values of the four parameters for each MARY profile were obtained by multi-Lorentzian fitting (Section S6 of the supplementary material).

Figure 3 plots the dependence of the four MARY parameters on $\left[\mathrm{AscH}_{2}\right]$ in the ranges $0.1-25 \mathrm{mM}(\mathrm{pH} \mathrm{3.0)}$ and $2-25 \mathrm{mM}(\mathrm{pH} 7.0)$ where the geminate radical pairs are largely triplet-born. All four quantities are larger at $\mathrm{pH} 3.0$ than at $\mathrm{pH}$ 7.0. At $\mathrm{pH} 3.0$, all four increase with increasing $\left[\mathrm{AscH}_{2}\right]$ in contrast to $\mathrm{pH} 7.0$ where they are independent of concentration.

At $\mathrm{pH} 7.0, B_{1 / 2}, B_{\mathrm{LFE}}, A_{\mathrm{LFE}}$, and $B_{\text {zero }}$ are all independent of $\left[\mathrm{AscH}_{2}\right]$ in the range $2-300 \mathrm{mM}$; the averaged values are given in Table I. After scaling to the same $M F E_{\text {sat }}$, the MARY profiles coincide to within experimental error across the whole range of concentrations (Fig. 2(d)).

At $\mathrm{pH} 3.0$, by contrast, the shape of the MARY profiles is strongly dependent on $\left[\mathrm{AscH}_{2}\right]$ (Fig. 2(c)) as the initial state of the radical pair changes from mostly triplet to mostly singlet. Table I gives the values of the four MARY parameters obtained by extrapolation of the data in Fig. 3 to $\left[\mathrm{AscH}_{2}\right]=0$. a

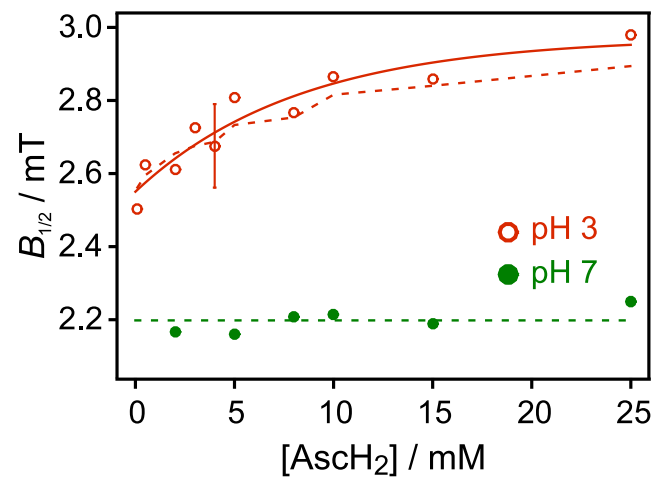

C

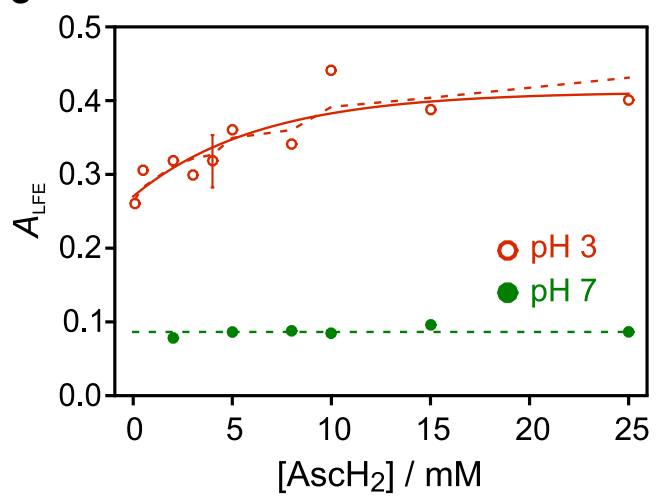

b

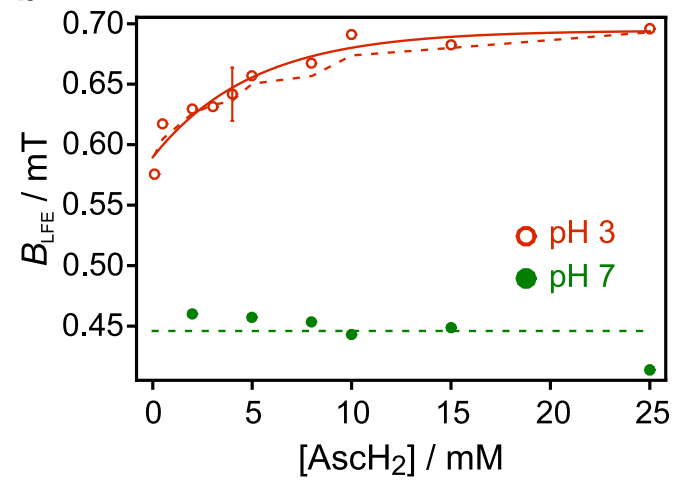

d

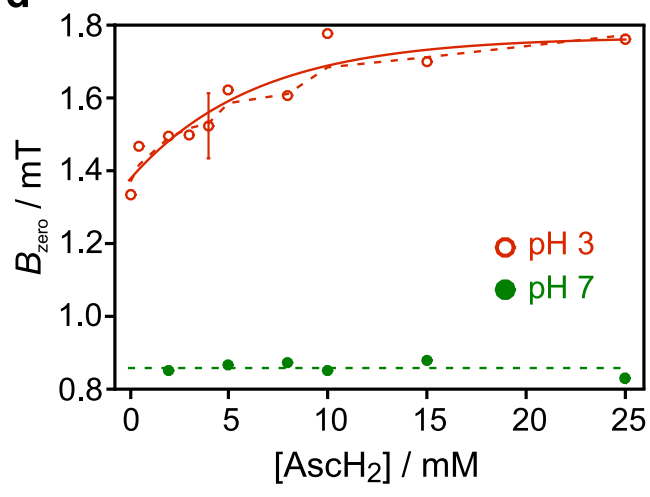

FIG. 3. Parameters extracted from MARY profiles for FMN $\left(10 \mu \mathrm{M}\right.$ ) with varying concentrations of $\mathrm{AscH}_{2}$ at $\mathrm{pH} 3.0$ (red, open circles) and $\mathrm{pH} 7.0$ (green, filled circles). (a) $B_{1 / 2}$, (b) $B_{\mathrm{LFE}}$, (c) $A_{\mathrm{LFE}}$, and (d) $B_{\text {zero. }}$ A representative $99 \%$ confidence interval is shown for $[$ AscH 2$]=5 \mathrm{mM}$ at $\mathrm{pH} 3.0$. The ionic strength was adjusted to $0.1 \mathrm{M}$ using appropriate concentrations of sodium chloride and sodium ascorbate. Green dashed lines indicate the average parameter values at $\mathrm{pH}$ 7.0. The red dashed lines show the parameter values at $\mathrm{pH} 3.0$ arising from the composite lineshape model discussed in the text. The solid red lines are

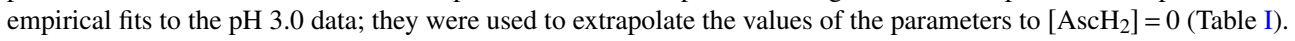


TABLE I. Characteristic MARY parameters for the $\mathrm{FMN}+\mathrm{AscH}_{2}$ reaction, extrapolated to $\left[\mathrm{AscH}_{2}\right]=0$.

\begin{tabular}{ccccc}
\hline \hline & $B_{1 / 2} / \mathrm{mT}$ & $B_{\mathrm{LFE}} / \mathrm{mT}$ & $B_{\text {zero }} / \mathrm{mT}$ & $A_{\mathrm{LFE}}$ \\
\hline pH 3.0 & $2.55 \pm 0.04$ & $0.59 \pm 0.01$ & $1.37 \pm 0.04$ & $0.27 \pm 0.02$ \\
pH 7.0 & $2.20 \pm 0.01$ & $0.45 \pm 0.01$ & $0.86 \pm 0.01$ & $0.09 \pm 0.01$ \\
\hline \hline
\end{tabular}

\section{E. $B_{1 / 2}$ values}

Ignoring any contribution from spin relaxation, a simple prediction of the width of the MARY profile is given by the Weller equation $^{28}$

$$
B_{1 / 2}=2 \frac{\left(B_{1}^{2}+B_{2}^{2}\right)}{B_{1}+B_{2}}
$$

in which $B_{1}$ and $B_{2}$ are effective hyperfine coupling constants for the two radicals,

$$
B_{i}=\left[\sum_{k} a_{i k}^{2} I_{k}\left(I_{k}+1\right)\right]^{1 / 2} .
$$

$I_{k}$ and $a_{i k}$ are, respectively, the spin quantum number and isotropic hyperfine coupling constant of nucleus $k$ in radical $i$ ( $i=1,2)$. Equation (2) is a good approximation for radical pairs of intermediate lifetime (when the hyperfine couplings are comparable to the reciprocal of the radical pair lifetime) and gives an upper bound for longer lived radical pairs. $^{9}$

At both $\mathrm{pH} 3.0$ and $\mathrm{pH}$ 7.0, the thermodynamically favoured protonation states of the radicals formed by electron transfer from $\mathrm{AscH}_{2}$ to $\mathrm{FMN}$ are $\mathrm{Asc}^{\circ-}$ and $\mathrm{FMNH}^{-}$(the $\mathrm{p} K_{\mathrm{a}} \mathrm{s}$ of $\mathrm{FMNH}_{2}{ }^{+}$and $\mathrm{FMNH}^{\cdot}$ are 2.3 and 8.3, respectively ${ }^{29}$ ). The transient absorption experiments (Section S3 of the supplementary material) show that these radicals are indeed formed at $\mathrm{pH}$ 3.0, as might be expected given that triplet FMN should be protonated at that $\mathrm{pH}$. The MARY profiles at $\mathrm{pH} 3.0$ should therefore be determined by the hyperfine interactions of $\mathrm{FMNH}^{-}$and $\mathrm{Asc}^{-}$.

At $\mathrm{pH} 7.0$, however, ${ }^{3} \mathrm{FMN}$ is not protonated and the initially formed anion radical, $\mathrm{FMN}^{--}$, was found to protonate with a time constant of $\sim 1 \mu$ s (Section S3 of the supplementary material). Since this is longer than the lifetime of the radical pair (estimated at $100 \mathrm{~ns}$ ), we can anticipate that the MARY profiles at $\mathrm{pH} 7.0$ should reflect the magnetic properties of $\mathrm{FMN}^{--}$and $\mathrm{Asc}^{\circ-}$.

These predictions seem to be confirmed by the close agreement of the experimental $B_{1 / 2}$ values with those obtained from Eq. (2) using literature values for the hyperfine couplings in $\mathrm{Asc}^{-}$(Ref. 24) and DFT values for the FMN radicals (Section $\mathrm{S} 1$ of the supplementary material). For the radical pair $\left[\mathrm{FMN}^{\cdot-} \mathrm{Asc}^{\cdot-}\right.$ ], Eq. (2) gives $B_{1 / 2}=2.20 \mathrm{mT}$ in exact agreement with the experimental value at $\mathrm{pH} 7.0$ (Table I). Turning to $\mathrm{pH} 3.0$, Eq. (2) gives $B_{1 / 2}=2.32 \mathrm{mT}$ for [FMNH ${ }^{*}$ $\mathrm{Asc}^{-}$], in fair agreement with the observed value, $2.55 \pm 0.04$ $\mathrm{mT}$ (Table I). The discrepancy between these estimates and the experimental value at $\mathrm{pH} 3.0$ cannot be attributed to a different protonation state of the ascorbyl radical: when combined with the experimental data for FMNH', the DFT-derived hyperfine couplings for $\mathrm{Asc}^{\cdot-}$, AscH ${ }^{\cdot}$ and $\mathrm{AscH}_{2}{ }^{++}$give $B_{1 / 2}=2.38 \mathrm{mT}$, $2.25 \mathrm{mT}$, and $2.39 \mathrm{mT}$, respectively. Thus, no clear assignment of the protonation state of the ascorbyl radical at $\mathrm{pH} 3.0$ is possible from the observed value of $B_{1 / 2}$.

\section{SPIN DYNAMICS SIMULATIONS}

To shed light on the factors that determine the size and shape of the LFE, we have simulated the spin dynamics of the reacting radical pairs. The charges of the FMN and ascorbyl radicals strongly affect their relative diffusion. Attractive Coulomb interactions are expected to enhance the effects of the inter-radical exchange interaction, which, though short-range, modifies the spin evolution of the diffusing radical pair and gives rise to singlet-triplet dephasing. ${ }^{30}$ This effect has been modelled in the past by introducing a spin-relaxation operator to account phenomenologically for the loss of singlet-triplet coherence. ${ }^{30-32}$ However, in doing so, one loses information on the microscopic details of the radical motion and its interplay with the spin evolution. Instead, we have aimed at a more complete description which relies on the direct solution of a stochastic Liouville equation for the distance-dependent spin density operator. Employing a generalized Green's function method, we have been able to obtain solutions for multinuclear spin systems of realistic complexity. ${ }^{33}$ The approach is valid if, during the time they remain spin-correlated, the diffusing radicals explore a configuration space much larger than the narrow range of radical-radical separations in which the exchange interaction and singlet recombination are effective. This condition is generally met for radical pairs in low viscosity, polar liquids, e.g., water at room temperature, in particular if all reverse electron transfer processes are in the Marcus normal region (and thus occur predominantly when the radicals are in contact). ${ }^{34,35}$ Green's function methods have proved to be efficient for tackling problems in spin chemistry, in particular for analytical studies of small model systems. ${ }^{33,36-43}$ Here, we employ the approach as a numerical tool to predict the recombination efficiency of realistic radical pairs, a task that, at low fields, is intractable by direct solution. For the convenience of the reader and to summarize the essence of the numerical implementation, we outline the theoretical fundamentals. Applications of the method have been documented by Doktorov and co-workers; readers interested in the theoretical details are referred to their work. ${ }^{33,36-43}$

\section{A. Theory}

In the experiments described here, the applied magnetic field alters the singlet recombination probability of the geminate radical pair, an effect detected via the change in the population of the fluorescent excited singlet state of FMN. The recombination probability depends on the initial spin configuration, the relative motion of the radicals, the rate constant of the spin-selective recombination reaction, and the spin dynamics that result from hyperfine, exchange, and Zeeman interactions. We model these factors by means of the electron-nuclear spin density operator, $\hat{\rho}(r, t)$, whose equation of motion, the stochastic Liouville equation, is

$$
\frac{\partial}{\partial t} \hat{\rho}(r, t)=\left(i \hat{\hat{L}}(r)+\hat{\mathcal{L}}(r)-\hat{\hat{K}}(r)-\tau_{\mathrm{sc}}^{-1}\right) \hat{\rho}(r, t) .
$$


The term involving the Liouvillian superoperator $\hat{\hat{L}}(r)$,

$$
\hat{\hat{L}}(r) \hat{\rho}(r, t)=\left(\hat{\hat{L}}_{0}+\hat{\hat{L}}_{\mathrm{ex}}(r)\right) \hat{\rho}(r, t)=-[\hat{H}(r), \hat{\rho}(r, t)],
$$

accounts for the coherent evolution under the spin Hamiltonian

$$
\hat{H}(r)=\hat{H}_{0,1}+\hat{H}_{0,2}-j(r)\left(2 \hat{\boldsymbol{S}}_{1} \cdot \hat{\boldsymbol{S}}_{2}+\frac{\hat{1}}{2}\right)
$$

in which

$$
\hat{H}_{0, i}=\omega_{0} \hat{S}_{i, z}+\sum_{k} a_{i, k} \hat{\boldsymbol{I}}_{i, k} \cdot \hat{\boldsymbol{S}}_{i}
$$

describes the interactions local to the individual radicals, i.e., Zeeman and hyperfine interactions ( $\hat{\hat{L}}_{0}$ in Eq. (5)), and $j(r)$, the exchange interaction $\left(\hat{\hat{L}}_{\mathrm{ex}}(r)\right.$ in Eq. (5)), which depends on the inter-radical distance, $r . \hat{\boldsymbol{S}}_{i}$ and $\hat{\boldsymbol{I}}_{i, k}$ are, respectively, electron and nuclear spin angular momentum operators; $a_{i, k}$ is the isotropic hyperfine coupling constant of nucleus $k$ in radical $i$; and $\omega_{0}$ is the strength of the electron Zeeman interaction.

The operator $\hat{\mathcal{L}}(r)$ in Eq. (4), which is diagonal in the Liouville spin-space, models the relative stochastic motion of the radicals and, for continuous diffusion, is given by

$$
\hat{\mathcal{L}}(r) \hat{\rho}(r, t)=\frac{D}{r^{2}} \frac{\partial}{\partial r} r^{2} \exp (-v(r)) \frac{\partial}{\partial r} \exp (+v(r)) \hat{\rho}(r, t),
$$

where $D$ is the mutual diffusion coefficient of the two radicals and $u(r)=v(r) k_{\mathrm{B}} T$ is the interaction potential, which for Coulombically interacting radicals is given by

$$
u(r)=k_{\mathrm{B}} T z_{1} z_{2} \frac{r_{\mathrm{c}}}{r}
$$

in which $z_{1}$ and $z_{2}$ are the charge numbers of the radicals and $r_{\mathrm{c}}$ is the Onsager radius for singly charged ions $\left(r_{\mathrm{c}}=7 \AA\right.$ in water at room temperature). Equation (4) obeys the reflective inner boundary condition at the contact distance of the two (spherical) radicals, $\sigma=r_{1}+r_{2}$,

$$
\begin{aligned}
\left.\hat{n}(r) \hat{\rho}(r, t)\right|_{r=\sigma}= & 4 \pi \sigma^{2} D \exp (-v(r)) \\
& \times\left.\frac{\partial}{\partial r} \exp (+v(r)) \hat{\rho}(r, t)\right|_{r=\sigma}=0,
\end{aligned}
$$

where $\hat{n}(r)$ is the flux operator.

The third term on the right-hand side of Eq. (4) accounts for radical recombination according to the Haberkorn approach, ${ }^{44}$

$$
\hat{\hat{K}}(r) \hat{\rho}(r, t)=\frac{1}{2} w_{\mathrm{S}}(r)\left[\hat{P}_{\mathrm{S}}, \hat{\rho}(r, t)\right]_{+},
$$

where $w_{\mathrm{S}}(r)$ is the distance-dependent recombination rate constant for the singlet state of the radical pair. Triplet recombination is negligible because the lowest locally excited triplet state has a higher free energy than the radical pair. Here and below, $\hat{P}_{i}(i=\mathrm{S}$ and $\mathrm{T})$ are the singlet and triplet projection operators. The $\tau_{\mathrm{sc}}$-term in Eq. (4) accounts for the non-spin-selective termination reactions of the radicals and, in an effective manner, for the loss of coherence by spin relaxation by removing radicals with lifetimes exceeding $\tau_{\mathrm{sc}}$. This approach allows larger spin systems to be treated than would typically be possible if an explicit superoperator for paramagnetic relaxation was employed.
Such an approximation is appropriate when, as here, the focus is on the shape of the MARY profile. Absolute product yields may be systematically affected if the recombination reaction occurs on a timescale comparable to or slower than $\tau_{\mathrm{sc}}$ (in which case a more complete theory accounting for F-pair processes would be required).

The quantum yield for formation of the singlet product is

$$
\begin{aligned}
\Phi_{\mathrm{S}} & =\operatorname{Tr}\left[\int_{0}^{\infty} \mathrm{d} t \int_{\sigma}^{\infty} \hat{\hat{K}}(r) \hat{\rho}(r, t) \mathrm{d}^{3} r\right] \\
& =\operatorname{Tr}\left[\hat{P}_{\mathrm{S}} \int_{0}^{\infty} \mathrm{d} t \int_{\sigma}^{\infty} w_{\mathrm{S}}(r) \hat{\rho}(r, t) \mathrm{d}^{3} r\right],
\end{aligned}
$$

which can be rewritten as

$$
\Phi_{\mathrm{S}}=\operatorname{Tr}\left[\hat{P}_{\mathrm{S}} \int_{\sigma}^{\infty} w_{\mathrm{S}}(r) \hat{\tilde{\rho}}(r, s=0) \mathrm{d}^{3} r\right],
$$

where the tilde denotes the Laplace transform, i.e., $\hat{\rho}(r, s)$ $=\int_{0}^{\infty} \hat{\rho}(r, t) \mathrm{e}^{-s t} \mathrm{~d} t$.

In general, the solution of Eq. (4) is non-trivial and can only be found by employing numerous approximations for all but the simplest spin systems. Here, we make use of the fact that the exchange interaction and the singlet recombination rate constant are rapidly decaying functions of the inter-radical distance and are only non-zero in a narrow range of separations close to the contact distance. In particular, the through-space model of the exchange interaction suggests that

$$
j(r)=j_{0} \exp \left(-r / r_{\mathrm{ex}}\right),
$$

with $r_{\text {ex }}$ in the range $0.34-0.50 \AA{ }^{45}$ Furthermore, for the radical pairs studied here, the singlet recombination reaction is in the Marcus "normal region" so that its distance dependence is controlled by the (square of the) coupling matrix element which has the same form as Eq. (14); ;6,47 the distancedependence of the reorganization energy and the reaction free energy are significantly weaker. ${ }^{34,35}$ Thus, both $j(r)$ and $w_{\mathrm{S}}(r)$ are expected to decay exponentially with distance and to be short range. In the experiments reported here, we focus on nonviscous aqueous solutions in which the radicals move rapidly and sample a large range of distances within the lifetime of the radical pair (or the spin coherence time, whichever is shorter). Under these conditions, the functional forms of $w_{\mathrm{S}}(r)$ and $j(r)$ (but certainly not their overall effect) are inconsequential and we may employ the contact approximation,

$j(r)=\frac{J}{4 \pi r \sigma} \delta(r-\sigma) \quad$ and $\quad w_{\mathrm{S}}(r)=\frac{k_{\mathrm{S}}}{4 \pi r \sigma} \delta(r-\sigma)$,

with the parameters $J$ and $k_{\mathrm{S}}$ chosen such that the integral strengths are equal to those of their $r$-dependent counterparts. The virtue of this approximation is that Eq. (4) can be solved using an elegant Green's function approach for the large spin systems studied here. For the first time, this method allows one to model the shape of the MARY curve of a multinuclear spin system subject to spin-selective recombination, diffusion in a Coulomb potential, and S/T-dephasing induced by modulation of the exchange interaction by stochastic motion.

It is convenient to define an operator $\hat{V}(r)$ that combines the rate and exchange operators,

$$
\hat{\hat{V}}(r)=i \hat{\hat{L}}_{\mathrm{ex}}(r)-\hat{\hat{K}}(r) \approx \hat{\hat{V}}_{0} \frac{\delta(r-\sigma)}{4 \pi r \sigma},
$$


where $\hat{\hat{V}}_{0}$ is the operator multiplier of the normalized deltafunction distribution in the contact approximation. If the radical pair is generated at the contact distance, $\sigma$ (as expected because the forward electron transfer occurs in the Marcus normal region, ${ }^{46,47}$ and the viscosity is low), the initial density operator is given by

$$
\hat{\rho}(r, t=0)=\frac{\delta(r-\sigma)}{4 \pi r \sigma} \hat{\rho}_{0},
$$

where $\hat{\rho}_{0}$ is typically the normalized singlet or triplet projection operator or a linear combination of the two. With the distance-dependent terms and the initial condition given by Eqs. (16) and (17), respectively, the Laplace transform of the density operator can be expressed in terms of the Green's superoperator $\hat{\hat{G}}\left(r \mid r_{0}, t\right)$, which is a generalization of a Green's function,

$$
\left(\frac{\partial}{\partial t}-\hat{\mathcal{L}}-i \hat{\hat{L}}_{0}\right) \hat{\hat{G}}\left(r \mid r_{0}, t\right)=\hat{\hat{1}} \frac{\delta\left(r-r_{0}\right)}{4 \pi r r_{0}} \delta(t),
$$

subject to the boundary condition $\left.\hat{n} \hat{\hat{G}}\left(r \mid r_{0}, t\right)\right|_{r=\sigma}=0$. Making use of the properties of Green's functions, ${ }^{48}$ it can be shown that

$$
\begin{aligned}
\hat{\tilde{\rho}}(\sigma, s=0)= & \left(\hat{\hat{1}}+\hat{\hat{\tilde{G}}}\left(\sigma \mid \sigma, s=\tau_{\mathrm{sc}}^{-1}\right) \hat{\hat{V}}_{0}\right)^{-1} \\
& \times \hat{\hat{G}}\left(\sigma \mid \sigma, s=\tau_{\mathrm{sc}}^{-1}\right) \hat{\rho}_{0},
\end{aligned}
$$

where, as above, the tilde denotes the Laplace transform (see Ref. 36 for details). For numerical purposes, it is convenient to recast Eq. (19) as

$$
\hat{\hat{\rho}}(\sigma, s=0)=\left(\hat{\hat{\tilde{G}}}^{-1}\left(\sigma \mid \sigma, s=\tau_{\text {sc }}^{-1}\right)+\hat{\hat{V}}_{0}\right)^{-1} \hat{\rho}_{0} .
$$

While Eq. (20) appears to be a trivial reformulation of Eq. (19), this transformation is crucial for the numerical solution of large spin systems. While, Eq. (19) cannot be solved easily for large spin systems, Eq. (20) preserves the block-structure, facilitating a more efficient solution. This is the central expression, which, in combination with Eq. (13), enables calculation of the singlet recombination yield of diffusing radicals with large numbers of nuclear spins. The benefit of the approach is that the Green's superoperator, $\hat{\hat{G}}\left(r \mid r_{0}, t\right)$, can be obtained from the Green's function of the underlying (scalar) diffusion problem, $g\left(r \mid r_{0}, t\right)$, according to ${ }^{33}$

$$
\hat{\hat{G}}\left(r \mid r_{0}, t\right)=g\left(r \mid r_{0}, t\right) \exp \left(i \hat{\hat{L}}_{0} t\right) .
$$

Here, $g\left(r \mid r_{0}, t\right)$ is the solution of

$$
\left(\frac{\partial}{\partial t}-\hat{\mathcal{L}}\right) g\left(r \mid r_{0}, t\right)=\frac{\delta\left(r-r_{0}\right)}{4 \pi r r_{0}} \delta(t),
$$

which, for the reflective boundary condition $\left.\hat{n} g\left(r \mid r_{0}, t\right)\right|_{r=\sigma}=0$, free diffusion (i.e., $r_{\mathrm{c}}=0$ ), and $r=r_{0}=\sigma$, is given by

$$
\tilde{g}^{-1}(\sigma \mid \sigma, s)=k_{\mathrm{D}}\left(1+\sqrt{s \tau_{\mathrm{d}}}\right),
$$

with $k_{\mathrm{D}}=4 \pi \sigma D$ and $\tau_{\mathrm{d}}=\sigma^{2} / D .^{37}$ In the presence of a Coulomb potential, an excellent approximation for $\tilde{g}(\sigma \mid \sigma, s)$ has been derived by Zharikov and Shokhirev. ${ }^{49}$ A more detailed elaboration of the approach is given in Ref. 33, where it is applied to derive an analytical solution of the recombination quantum yield for a one-nucleus (spin-1/2) radical pair at zero-field. Section S7 of the supplementary material shows how $\Phi_{\mathrm{S}}$ (Eq. (13)) is related to the magnetic field effect on the fluorescence intensity under conditions of continuous illumination.

\section{B. Simulations}

The theoretical framework outlined above was used to obtain the dependence of the MARY parameters defined in Fig. 1(b) $\left(B_{1 / 2}, B_{\mathrm{LFE}}, B_{\text {zero }}\right.$, and $\left.A_{\mathrm{LFE}}\right)$ on the strength of the exchange interaction in the range $-1500<J / \AA^{3} \mathrm{~ns}^{-1}<1500$. The contact distance of the radical pair was taken as $\sigma=7 \AA$, and the mutual diffusion coefficient $\left(D=129 \AA^{2} \mathrm{~ns}^{-1}\right)$ was calculated using the Stokes-Einstein law with a stick boundary condition. Other parameters were $k_{\mathrm{S}}=1.6 \times 10^{4} \AA^{3} \mathrm{~ns}^{-1}$ (typical for a diffusion controlled reaction in a non-viscous solvent) and $\tau_{\mathrm{sc}}=500 \mathrm{~ns}$ (a typical spin relaxation time). The hyperfine interactions were appropriate for the $\mathrm{FMN}^{-}$and Asc $^{--}$radicals (Section S1 of the supplementary material).

Figure 4 shows such calculations for radical pairs with charge numbers: $-3 \leq z_{1} z_{2} \leq 3$. Two features stand out. First, all four of the MARY parameters, $B_{1 / 2}, B_{\mathrm{LFE}}, B_{\text {zero }}$, and $A_{\mathrm{LFE}}$, are strongly influenced by the charges. Attractive Coulomb interactions $\left(z_{1} z_{2}<0\right)$ increase the recombination probability and reduce the lifetime of the radical pair causing all four parameters to increase relative to the case of free diffusion $\left(z_{1} z_{2}=0\right)$. Second, for repulsive Coulomb interactions $\left(z_{1} z_{2}>0\right)$, the exchange interaction has a much smaller effect on the shape of the MARY profile. Identical calculations (with $-3 \leq z_{1} z_{2} \leq 3$ ) but using the hyperfine interactions of $\mathrm{FMNH}^{-}$instead of $\mathrm{FMN}^{--}$gave similar results (Section S8 of the supplementary material). The implications of Fig. 4 are discussed in detail below.

\section{DISCUSSION}

\section{A. Magnetic field effects at $\mathrm{pH} 7.0$}

In principle, either of the radical pairs $\left[\mathrm{FMN}^{--} \mathrm{AscH}{ }^{-}\right]$ and $\left[\mathrm{FMN}^{-\cdot} \mathrm{Asc}^{--}\right]$could be responsible for the $\mathrm{pH} 7.0$ data in Fig. 3. At pH 7.0, ascorbic acid exists as the ascorbate ion, $\mathrm{AscH}^{-}$, so that the neutral $\mathrm{AscH}^{\cdot}$ radical would be formed by electron transfer to photoexcited FMN. AscH ${ }^{*}$ is highly acidic $\left(\mathrm{p} K_{\mathrm{a}}=-0.45^{24}\right)$ and should deprotonate to form $\mathrm{Asc}^{\cdot-}$. In fact, in many cases, ascorbate reacts by hydrogen atom transfer, forming Asc ${ }^{--}$directly. ${ }^{50}$ The transient absorption experiments (Section S3 of the supplementary material) cannot distinguish the two pathways nor do they help decide which of $\mathrm{AscH}^{\circ}$ and $\mathrm{Asc}^{--}$is likely to be present during the period ( 1-100 ns) when the MFE is generated.

The charges of the radicals have a strong effect on the MARY parameters (Fig. 4). The major form of the flavin radical at $\mathrm{pH} 7.0\left(\mathrm{FMN}^{-}, 86 \%\right)$ has charge $z_{1}=-3$ (with the phosphate group present as $\mathrm{R}-\mathrm{OPO}_{3}^{2-}$ ). The ascorbyl radicals, $\mathrm{AscH}{ }^{-}$and $\mathrm{Asc}^{--}$, have charges $z_{2}=0$ and $z_{2}=-1$, respectively. Therefore, $z_{1} z_{2}=0$ for $\left[\mathrm{FMN}^{-} \mathrm{AscH}^{-}\right]$and $z_{1} z_{2}=+3$ for $\left[\mathrm{FMN}^{--}\right.$Asc $\left.^{--}\right]$. The former should undergo 
a

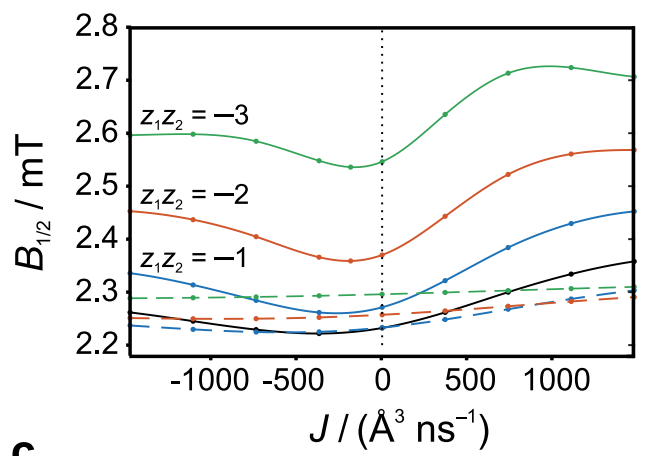

C

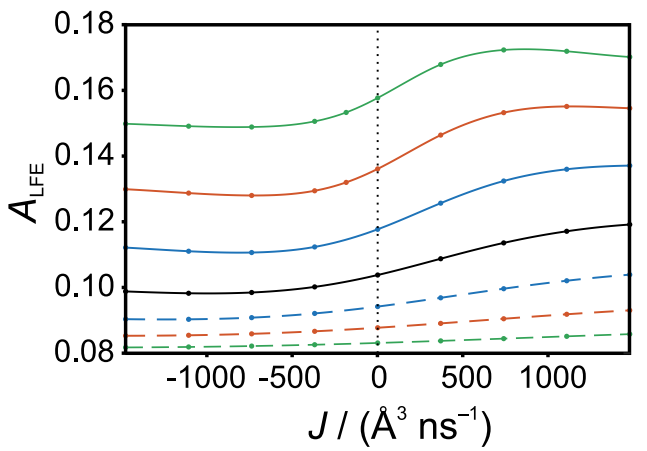

b
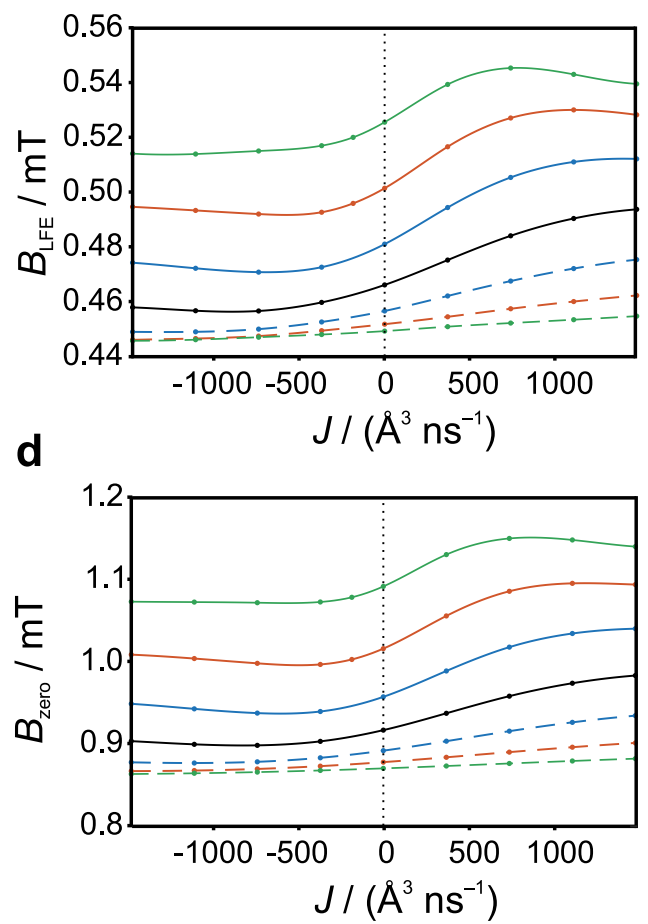

FIG. 4. Calculated dependence of the MARY parameters on the strength of the contact exchange interaction and the charges of the radicals, using hyperfine interactions appropriate for $\mathrm{FMN}^{--}$and $\mathrm{Asc}^{--}$(Section S1 of the supplementary material). The solid lines are for attractive (green: $z_{1} z_{2}=-3$, orange: $z_{1} z_{2}=-2$, blue: $z_{1} z_{2}=-1$ ) or no Coulomb interactions (black); the dashed lines are for repulsive interactions, with the same colour coding. Additional parameters: $k_{\mathrm{S}}=1.6 \times 10^{4} \AA^{3} \mathrm{~ns}^{-1}, \tau_{\mathrm{sc}}=500 \mathrm{~ns}, r_{\mathrm{c}}=7 \AA, D=129 \AA^{2} \mathrm{~ns}^{-1}, \sigma=7 \AA$. The values of the parameters are independent of the initial spin configuration.

free diffusion while the latter should be strongly affected by the repulsive Coulomb interaction. Markedly different MARY profiles can be expected.

Exploratory simulations of the MARY profiles for $\left[\mathrm{FMN}^{--} \mathrm{Asc}^{--}\right.$(Section S9 of the supplementary material, Fig. S9.1) confirm the prediction that the MFEs for singlet and triplet initial spin configurations should have identical shapes and opposite signs (as also predicted from model calculations for radical pairs comprising a single spin $-1 / 2$ nucleus $^{51}$ and for radical pairs at high field ${ }^{41}$ ), as found experimentally (Figs. 2(b) and 2(d)). The four MARY parameters extracted from these profiles are in broad agreement with the experimental values (Table I) when the effective relaxation time $\tau_{\mathrm{sc}}$ (Eq. (4)) is in the physically reasonable range, $500 \mathrm{~ns}$ to $2 \mu \mathrm{s}$. The simulations are only weakly sensitive to the integral strength of the singlet recombination rate constant, $k_{\mathrm{S}}$, and to the presence of an exchange interaction. By contrast, simulations for the freely diffusing $\left[\mathrm{FMN}^{--} \mathrm{AscH}{ }^{\cdot}\right]$ radical pair (for which $z_{1} z_{2}=0$, Section $\mathrm{S} 9$ of the supplementary material, Fig. S9.2) cannot account for the observed MARY parameters. We conclude that either $\mathrm{AscH}^{\cdot}$ is initially formed and deprotonates so rapidly that it does not contribute to the MARY profile or that it is not formed at all, i.e., the reaction mechanism is hydrogen atom transfer rather than electron transfer followed by deprotonation. It is remarkable that the MFE data allow such reaction details to be determined, a task that would be very challenging using optical spectroscopy.

We have also formulated a surrogate model ${ }^{52,53}$ to determine $k_{\mathrm{S}}$ and $k_{\mathrm{sc}}$ from the four MARY parameters. The best-fit values of $k_{\mathrm{S}}$ and $k_{\mathrm{sc}}$ are given in Table II.

\section{B. Magnetic field effects at $\mathrm{pH} 3.0$}

In contrast to $\mathrm{pH} 7.0$, the shape of the MARY profile at $\mathrm{pH}$ 3.0 depends on the ascorbic acid concentration (Fig. 2(c)). As the concentration is increased, the MFE broadens and inverts and the LFE disappears. This behaviour cannot be attributed to degenerate proton-coupled electron exchange (between $\mathrm{AscH}^{-}$and $\mathrm{Asc}^{--}$) because the rate constant ${ }^{54}$ is too small for there to be an effect on a 1-100 ns timescale at ascorbic acid concentrations in the range studied here $(\leq 300 \mathrm{mM})$.

The MARY data shown in Fig. 2(c) were fitted globally to a model in which the profile for each $\mathrm{AscH}_{2}$ concentration was represented as a weighted sum of profiles for two chemically distinct radical pairs, one born in a singlet state, the other born in a triplet state. The profiles obtained by this procedure are shown in Fig. 5. Each profile was modelled as a sum of Lorentzian lines (Section S6 of the supplementary material) from which values of the four MARY parameters were extracted (see Table II). The triplet signal $\left(B_{1 / 2}=2.56 \mathrm{mT}\right.$, $B_{\mathrm{LFE}}=0.59 \mathrm{mT}$ ) which dominates at low ascorbic acid concentrations gradually gives way to the singlet signal $\left(B_{1 / 2}=2.31 \mathrm{mT}\right.$ and $\left.B_{\mathrm{LFE}}=0.47 \mathrm{mT}\right)$ as the concentration is increased (and singlet electron transfer becomes faster than intersystem crossing, Fig. 1(a)). The disappearance of the LFE as $\left[\mathrm{AscH}_{2}\right]$ approaches $300 \mathrm{mM}$ results from cancellation of the opposite-phase signals (Fig. 5). The broadening of the MARY profile in the range $0 \leq\left[\mathrm{AscH}_{2}\right] \leq 25 \mathrm{mM}$ (Fig. 3(a)) also arises from the superposition of the two contributions. In agreement with Fig. 3, $B_{1 / 2}$ and $B_{\mathrm{LFE}}$ for both radical pairs are substantially larger than the corresponding values at $\mathrm{pH} 7.0$ 
TABLE II. Model fitting of magnetic field effects.

\begin{tabular}{|c|c|c|c|c|c|c|}
\hline & \multicolumn{2}{|c|}{$\mathrm{pH} 3.0$, species 1} & \multicolumn{2}{|c|}{$\mathrm{pH} 3.0$, species 2} & \multicolumn{2}{|c|}{$\mathrm{pH} 7.0$} \\
\hline Model & \multicolumn{2}{|l|}{ Bound } & \multicolumn{2}{|c|}{ Bound } & \multicolumn{2}{|c|}{ Diffusing, $z_{1} z_{2}=+3$} \\
\hline Radicals & \multicolumn{2}{|c|}{$\mathrm{FMNH}^{\cdot}+\mathrm{Asc}^{\cdot-}$} & \multicolumn{2}{|c|}{$\mathrm{FMNH}^{\cdot}+\mathrm{Asc}^{\cdot-}$} & \multicolumn{2}{|c|}{$\mathrm{FMN}^{--}+\mathrm{Asc}^{--}$} \\
\hline Parameters & \multicolumn{2}{|c|}{$k_{\mathrm{S}}=2.0 \times 10^{8} \mathrm{~s}^{-1}$} & \multicolumn{2}{|c|}{$k_{\mathrm{S}}=1.0 \times 10^{8} \mathrm{~s}^{-1}$} & \multicolumn{2}{|c|}{$k_{\mathrm{S}}=7.8 \times 10^{4} \AA^{3} \mathrm{~ns}^{-1}$} \\
\hline & \multicolumn{2}{|c|}{$k_{\mathrm{esc}}=2.5 \times 10^{7} \mathrm{~s}^{-1}$} & \multicolumn{2}{|c|}{$k_{\mathrm{esc}}=8.7 \times 10^{6} \mathrm{~s}^{-1}$} & \multicolumn{2}{|c|}{$k_{\mathrm{sc}}=1.0 \times 10^{6} \mathrm{~s}^{-1}$} \\
\hline \multirow[b]{2}{*}{ Initial state } & \multicolumn{2}{|c|}{ Triplet } & \multicolumn{2}{|c|}{ Singlet } & \multicolumn{2}{|c|}{ Either } \\
\hline & Exp. $^{a}$ & Calc. $^{\text {b }}$ & Exp. & Calc. & Exp. & Calc. \\
\hline$B_{1 / 2} / \mathrm{mT}$ & 2.56 & 2.59 & 2.31 & 2.29 & 2.20 & 2.25 \\
\hline$B_{\text {zero }} / \mathrm{mT}$ & 1.37 & 1.36 & 1.03 & 0.99 & 0.86 & 0.85 \\
\hline$B_{\mathrm{LFE}} / \mathrm{mT}$ & 0.59 & 0.59 & 0.47 & 0.44 & 0.45 & 0.43 \\
\hline$A_{\mathrm{LFE}}$ & 0.33 & 0.31 & 0.21 & 0.21 & 0.09 & 0.08 \\
\hline
\end{tabular}

(Table II). Before commenting on the possible identities of the two radical pairs, we first discuss our attempts to simulate the MARY profiles of the two radical pairs.

\section{Radical pair complex at $\mathrm{pH} 3.0$}

All four of the MARY parameters plotted in Fig. 3 are significantly larger at $\mathrm{pH} 3.0$ than at $\mathrm{pH}$ 7.0. For instance, when extrapolated to $\left[\mathrm{AscH}_{2}\right]=0$, the LFE at $\mathrm{pH} 3.0$ is $27 \%$ of $M F E_{\text {sat }}$ compared to $9 \%$ at $\mathrm{pH} 7.0$ (Table I). The simulations in Fig. 4 suggest that this could result from attractive Coulomb interactions which would increase the re-encounter probability thereby increasing the influence of the exchange interaction and potentially boosting the LFE and broadening the MARY profile.

As described above, the radical form of FMN at $\mathrm{pH}$ 3.0 is expected to be $\mathrm{FMNH}^{\cdot}$ which has charge $z_{1}=-1$ (from the phosphate group, $\mathrm{R}-\mathrm{OP}(\mathrm{OH}) \mathrm{O}_{2}^{-}$). The only way to achieve an attractive Coulomb potential would be if the ascorbyl radical was doubly protonated (i.e., $\mathrm{AscH}_{2}{ }^{++}$, $\left.z_{2}=+1\right)$ throughout the time required to generate the MFE (1-100 ns). The more stable, and more likely forms, $\mathrm{AscH}^{\cdot}$ $\left(z_{2}=0\right)$ and $\operatorname{Asc}^{--}\left(z_{2}=-1\right)$, would have, respectively, no

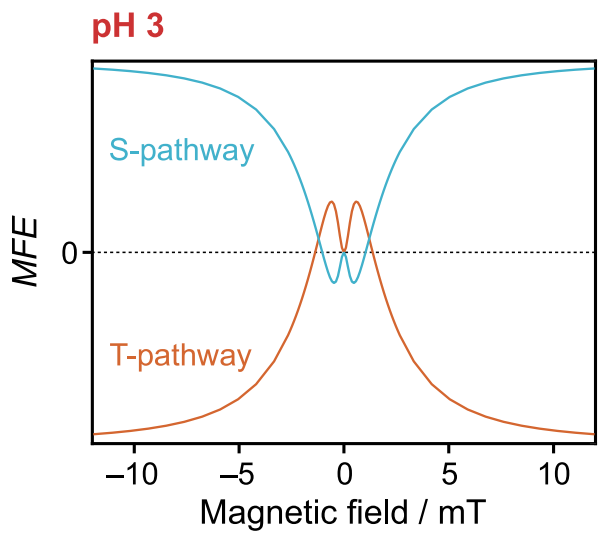

FIG. 5. MARY profiles of singlet- and triplet-born radical pairs at $\mathrm{pH}$ 3.0. The lineshapes were determined by simultaneously decomposing the concentration-dependent MARY profiles in Fig. 2(c).
Coulomb interaction and a repulsive Coulomb interaction with FMNH'. Simulations for [FMNH' $\left.\mathrm{AscH}_{2}{ }^{-+}\right]\left(z_{1} z_{2}=-1\right)$ do not reproduce the large LFE observed for $\left[\mathrm{AscH}_{2}\right]<25 \mathrm{mM}$ and suggest that $z_{1} z_{2}$ would need to be more negative than -3 to account for the observed MARY profile (Section S10 of the supplementary material). This holds true whichever set of hyperfine coupling constants is used (EPR- or DFT-derived, for all of the various possible radical protonation states). Even when (hypothetically) there are no hyperfine interactions in the ascorbyl radical (a situation expected to maximise the LFE amplitude), the model only matches the experimental LFE when $z_{1} z_{2}=-3$ is used (Section S10 of the supplementary material). No other combination of parameter values in the above model could be found that would account for the data at $\mathrm{pH}$ 3.0. In short, it would appear that a significant attractive interaction is required but that it cannot be simply Coulombic.

In an attempt to account for the experimental data at $\mathrm{pH}$ 3.0, we have simulated MARY profiles for weakly bound radical pairs with a fixed inter-radical distance. The spin dynamics were calculated using

$$
\frac{\partial}{\partial t} \hat{\rho}(t)=\left(i \hat{\hat{L}}-\hat{\hat{K}}-\tau_{\text {esc }}^{-1}\right) \hat{\rho}(t)
$$

where $\hat{\hat{L}}$ accounts for the coherent spin evolution arising from hyperfine and Zeeman interactions and, optionally, the exchange interaction within the complex. The $\tau_{\text {esc }}\left(=k_{\text {esc }}^{-1}\right)$ term describes the decay of the radical pair population by processes other than geminate recombination. As before, the radical pair was assumed to recombine exclusively via the singlet state. While the diffusion model gives rise to recombination kinetics with a $t^{-3 / 2}$ asymptote, the complexation model is characterised by exponential kinetics. ${ }^{5}$ Similar models have been used in the past for intramolecular radical pairs and radical pairs incorporated into micelles. ${ }^{55}$ Moreover, there is a precedent for a (triplet) flavin-ascorbic acid complex, postulated in the context of triplet quenching. ${ }^{27}$

Figure 6 summarizes the results of such calculations for the radical pair $\left[\mathrm{FMNH}^{-} \mathrm{Asc}^{--}\right]$in the absence of exchange interactions. No preference for the protonation state of the ascorbyl radical is implied by this choice; very similar results were obtained for $\mathrm{AscH}^{\cdot}$ and $\mathrm{AscH}_{2}{ }^{++}$. By systematically 
a

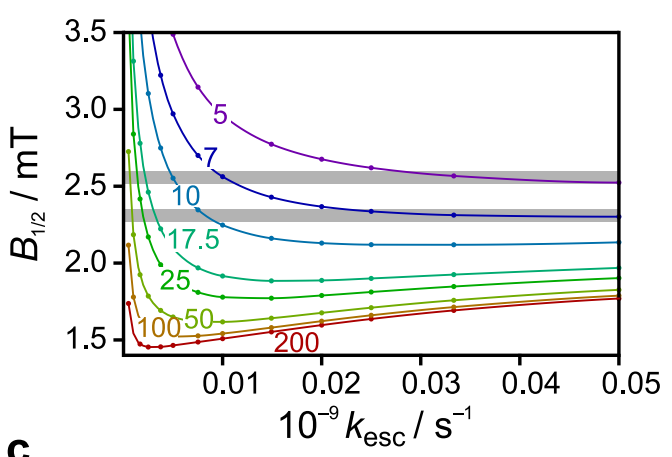

C

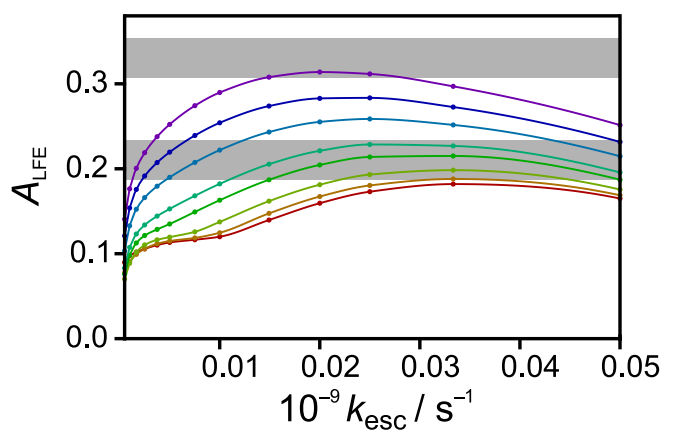

b

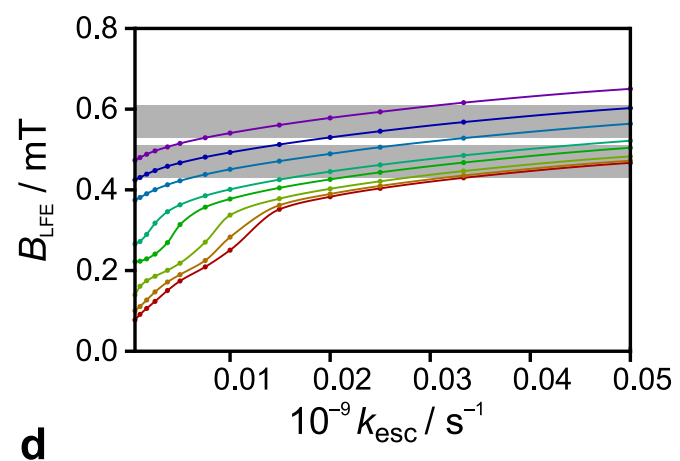

d

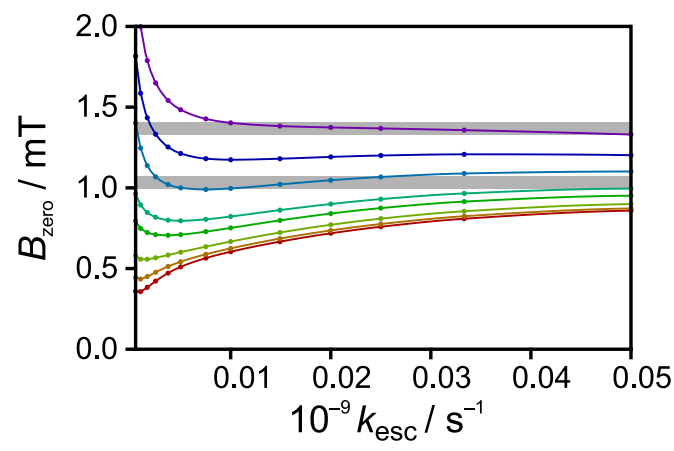

FIG. 6. Calculated dependence of the MARY parameters on the escape rate constant $k_{\text {esc }}$ for a range of values of $k_{\mathrm{S}}$ using hyperfine interactions appropriate for FMNH and Asc ${ }^{--}$. The values of $\left(k_{\mathrm{S}}\right)^{-1}$ in nanoseconds are given in the top left panel; the same colour coding has been used for the other panels. The grey horizontal bars indicate the experimentally determined parameters for the triplet- and the singlet-born radical pairs at $\mathrm{pH}$ 3.0. The best-fitting parameter sets for the two radical pairs are reported in Table II.

varying $k_{\text {esc }}$ and $k_{\mathrm{S}}$ (the singlet recombination rate constant), it was found that the experimental observations are consistent with $k_{\mathrm{S}} \approx 1-2 \times 10^{8} \mathrm{~s}^{-1}$ and $k_{\mathrm{esc}} \approx 10^{7} \mathrm{~s}^{-1}$ and that an exchange interaction is not required to account for the data. To obtain estimates of the MARY parameters, surrogate models $^{52,53}$ were constructed to match the experimental parameters (determined from the above decomposition of the MARY profiles) to the predictions of the model (in which the two radicals exist as a weakly bound complex) using the sum of squared relative differences of the four MARY parameters to judge the goodness of the fit. The results are given in Table II.

To estimate how strong the binding interaction would need to be, we have simulated the diffusive escape of particles from square-well potentials (Section S11 of the supplementary material). For the triplet-born pair, we find that an interaction energy of $6.2 k_{\mathrm{B}} T$ (or $6.7 k_{\mathrm{B}} T$ when a repulsive Coulomb interaction, $z_{1} z_{2}=+1$, is included) is consistent with $k_{\mathrm{esc}}=2.5 \times 10^{7} \mathrm{~s}^{-1}$. For the singlet-born pair, an interaction energy of $7.3 k_{\mathrm{B}} T$ (or $7.7 k_{\mathrm{B}} T$ ) was found for $k_{\mathrm{esc}}=8.7 \times 10^{6} \mathrm{~s}^{-1}$. Such small changes in the energies of the radicals, which could be realised by hydrogen bonding, are not expected to give rise to significant changes in the optical spectra of bound FMN relative to its free form.

Neither fluorescence nor absorption spectra provide direct evidence of a ground state complex, in agreement with previous reports. ${ }^{27} \mathrm{We}$ envisage that the complex is formed after the electron is transferred resulting in a bound radical pair able to undergo efficient reverse electron transfer, as suggested by the large value of $k_{\mathrm{S}}$ obtained from the simulations $\left(1.0-2.0 \times 10^{8} \mathrm{~s}^{-1}\right.$, Table II).

We have assessed the effect of exchange interactions on the spin evolution of the bound radical pair (Section S12 of the supplementary material). Average exchange interactions exceeding $5 \mathrm{MHz}$ in absolute value have a marked effect on the MARY parameters and make it impossible to simulate the experimental data (Section S13 of the supplementary material). As well as this static effect, the exchange interaction can induce singlet-triplet dephasing when the inter-radical distance within the complex is modulated by thermal motions. We find that dephasing rate constants of up to approximately $10^{7} \mathrm{~s}^{-1}$ have a negligible effect on both $B_{1 / 2}$ and the shape of the LFE. Larger dephasing rates give rise to broadening of the MARY spectra and increased LFE amplitudes (Section S13 of the supplementary material). A simple model calculation (Section S12 of the supplementary material) suggests that both the average exchange interaction and the dephasing caused by stochastic modulation could be small enough as to have a negligible effect on the MARY profiles. This is particularly true for complexes resulting from the interaction of the ascorbyl radical with the phosphate group of the FMN, a situation similar to that of the intramolecular radical pair derived from FAD, for which no pronounced effects of the exchange interaction have been apparent in recent studies. ${ }^{13,56,57}$

It is clear that the two radical pairs, one singlet, the other triplet, differ in terms of their hyperfine interactions and/or their kinetics. Their identities are uncertain but it seems possible that they could differ in their protonation states. A 
more detailed study will be needed to clarify the detailed chemistry.

\section{CONCLUSIONS}

Radical pairs produced by electron transfer from ascorbic acid to photo-excited FMN exhibit magnetic field effects that peak at applied magnetic fields only a factor of ten stronger than the Earth's magnetic field. Relative to the effects at high field, the amplitudes of the LFEs observed here exceed those previously reported for intermolecular electron donoracceptor systems in aqueous solution, including biologically relevant molecules such as cryptochromes (the putative magnetoreceptor of the magnetic compass sense in migratory birds). ${ }^{12,13}$ This was unexpected because the reaction is formally bimolecular and thus subject to diffusion effects which normally result in small LFEs. The large effects arise, in part, from the asymmetric distribution of the hyperfine couplings in the two radicals: ${ }^{9}$ the ascorbyl radical has only one significant hyperfine interaction ${ }^{24}$ while the FMN radical has several. The prominent LFEs are also a product of attractive forces between the radicals arising from either Coulomb or specific interactions, as evidenced here by the $\mathrm{pH}-$ dependence of the magnetic field effects. In acidic solution, the formation of a transient weakly bound complex causes the LFE to reach an amplitude of $27 \%$ (relative to the MFE at high field), three times larger than at neutral $\mathrm{pH}$ where the radicals are freely diffusing or subject to repulsive Coulomb interactions.

The reactions can be efficiently modelled by a stochastic Liouville equation assuming that the radical pairs either diffuse in a Coulomb potential or are weakly bound and recombine with exponential kinetics. Employing a powerful Green's function method, we have simulated diffusing radical pairs with large numbers of coupled nuclei, undergoing spin-selective recombination reactions and singlet-triplet dephasing. For diffusing radicals, the LFE is surprisingly invariant to the spin-dephasing induced by modulation of the exchange interaction by the stochastic motion of the radicals.

The shape of the magnetic field effect at $\mathrm{pH} 7.0$ is independent of the ascorbic acid concentration and its phase is determined by the relative rates of the electron transfer reaction and the intersystem crossing of the photo-excited FMN. The invariance of the shape indicates that the same transient radical pair, $\left[\mathrm{FMN}^{--} \mathrm{Asc}^{--}\right]$, is produced by the singlet and triplet pathways. The repulsive Coulomb interaction between the radicals accounts for the remarkably inefficient singlet-triplet dephasing caused by modulation of the electron-electron exchange interaction by diffusive re-encounters. This is the first time that otherwise identical singlet and triplet-born radical pairs have been studied in the same reaction system. Assignment of the protonation state of the ascorbyl radical is difficult to determine spectroscopically but can be inferred from the MFE profile.

The large LFEs observed at $\mathrm{pH} 3.0$ cannot be rationalized in terms of the radicals diffusing in an attractive Coulomb potential. Instead, the data are consistent with a bound radical pair with lifetime of $\sim 100 \mathrm{~ns}$. There is no evidence of complex formation from the optical spectra at $\mathrm{pH} 3.0$ suggesting that the association is specific to the radical pair state. In contrast to $\mathrm{pH}$ 7.0, the MARY profiles vary strongly with the ascorbic acid concentration reflecting the different proportions of two chemically distinct radical pairs which probably differ in their protonation states and reactivity. The sensitivity to weak magnetic fields results not only from large intrinsic LFEs but also from the superposition of two slightly different MARY profiles with opposite sign. The largest apparent LFE is observed for intermediate ascorbic acid concentrations and amounts to $40 \%$ of the saturating MFE. At larger ascorbic acid concentrations, the effect changes sign and yields an atypical composite lineshape with significant contributions from both singlet- and triplet-born radical pairs.

\section{SUPPLEMENTARY MATERIAL}

See supplementary material for EPR, transient absorption, fluorescence quenching data, and additional calculations and simulations.

\section{ACKNOWLEDGMENTS}

E.W.E. thanks the Engineering and Physical Sciences Research Council (EPSRC) and SABMiller plc for a doctoral scholarship. We are grateful to the following for financial support: the European Research Council (under the European Union's 7th Framework Programme, No. FP7/20072013/ERC Grant Agreement No. 340451), the Air Force Office of Scientific Research (Air Force Materiel Command, USAF Award No. FA9550-14-1-0095), the EMF Biological Research Trust, and the EPSRC (Grant No. EPL011972/1). We are grateful to Dr. W. K. Myer for useful discussions.

${ }^{1}$ K. M. Salikhov, Y. N. Molin, R. Z. Sagdeev, and A. L. Buchachenko, Spin Polarization and Magnetic Field Effects in Radical Reactions (Elsevier, New York, 1984).

${ }^{2}$ U. E. Steiner and T. Ulrich, Chem. Rev. 89, 51 (1989).

${ }^{3}$ P. J. Hore and H. Mouritsen, Annu. Rev. Biophys. 45, 299 (2016)

${ }^{4}$ I. Lagroye, Y. Percherancier, J. Juutilainen, F. Poulletier De Gannes, and B. Veyret, Prog. Biophys. Mol. Biol. 107, 369 (2011).

${ }^{5}$ C. R. Timmel, U. Till, B. Brocklehurst, K. A. McLauchlan, and P. J. Hore, Mol. Phys. 95, 71 (1998).

${ }^{6}$ B. Brocklehurst, J. Chem. Soc., Faraday Trans. 2 72, 1869 (1976).

${ }^{7}$ K. Maeda, K. B. Henbest, F. Cintolesi, I. Kuprov, C. T. Rodgers, P. A. Liddell, D. Gust, C. R. Timmel, and P. J. Hore, Nature 453, 387 (2008).

${ }^{8}$ A. Weller, H. Staerk, and R. Treichel, Faraday Discuss. 78, 271 (1984).

${ }^{9}$ C. T. Rodgers, S. A. Norman, K. B. Henbest, C. R. Timmel, and P. J. Hore, J. Am. Chem. Soc. 129, 6746 (2007).

${ }^{10}$ T. D. Nguyen, B. R. Gautam, E. Ehrenfreund, and Z. V. Vardeny, Phys. Rev. Lett. 105, 166804 (2010).

${ }^{11}$ T. D. Nguyen, G. Hukic-Markosian, F. J. Wang, L. Wojcik, X. G. Li, E. Ehrenfreund, and Z. V. Vardeny, Nat. Mater. 9, 345 (2010).

${ }^{12}$ K. Maeda, A. J. Robinson, K. B. Henbest, H. J. Hogben, T. Biskup, M. Ahmad, E. Schleicher, S. Weber, C. R. Timmel, and P. J. Hore, Proc. Natl. Acad. Sci. U. S. A. 109, 4774 (2012).

${ }^{13}$ J. P. Beardmore, L. M. Antill, and J. R. Woodward, Angew. Chem. 54, 8494 (2015).

${ }^{14}$ D. R. Kattnig, E. W. Evans, V. Déjean, C. A. Dodson, M. I. Wallace, S. R. Mackenzie, C. R. Timmel, and P. J. Hore, Nat. Chem. 8, 384 (2016).

${ }^{15}$ A. A. Lee, J. C. S. Lau, H. J. Hogben, T. Biskup, D. R. Kattnig, and P. J. Hore, J. R. Soc., Interface 11, 20131063 (2014).

${ }^{16}$ T. Ritz, S. Adem, and K. Schulten, Biophys. J. 78, 707 (2000). 
${ }^{17}$ IARC, Monographs on the Evaluation of Carcinogenic Risks to Humans (International Agency for Research on Cancer, Lyon, 2002), Vol. 80.

${ }^{18}$ NRPB, Report of an Advisory Group on Non-ionising Radiation. Documents of the NRPB No. 1 (National Radiological Protection Board, Chilton, Oxon, UK, 2001), Vol. 12.

${ }^{19}$ C. T. Rodgers, Pure Appl. Chem. 81, 19 (2009).

${ }^{20}$ E. W. Evans, J. Li, J. G. Storey, K. Maeda, K. B. Henbest, C. A. Dodson, P. J. Hore, S. R. Mackenzie, and C. R. Timmel, Phys. Chem. Chem. Phys. 17, 18456 (2015)

${ }^{21}$ K. B. Henbest, K. Maeda, P. J. Hore, M. Joshi, A. Bacher, R. Bittl, S. Weber, C. R. Timmel, and E. Schleicher, Proc. Natl. Acad. Sci. U. S. A. 105, 14395 (2008).

${ }^{22}$ M. J. Frisch, G. W. Trucks, H. B. Schlegel, G. E. Scuseria, M. A. Robb, J. R. Cheeseman, G. Scalmani, V. Barone, B. Mennucci, G. A. Petersson, H. Nakatsuji, M. Caricato, X. Li, H. P. Hratchian, A. F. Izmaylov, J. Bloino, G. Zheng, J. L. Sonnenberg, M. Hada, M. Ehara, K. Toyota, R. Fukuda, J. Hasegawa, M. Ishida, T. Nakajima, Y. Honda, O. Kitao, H. Nakai, T. Vreven, J. A. Montgomery, Jr., J. E. Peralta, F. Ogliaro, M. J. Bearpark, J. Heyd, E. N. Brothers, K. N. Kudin, V. N. Staroverov, R. Kobayashi, J. Normand, K. Raghavachari, A. P. Rendell, J. C. Burant, S. S. Iyengar, J. Tomasi, M. Cossi, N. Rega, N. J. Millam, M. Klene, J. E. Knox, J. B. Cross, V. Bakken, C. Adamo, J. Jaramillo, R. Gomperts, R. E. Stratmann, O. Yazyev, A. J. Austin, R. Cammi, C. Pomelli, J. W. Ochterski, R. L. Martin, K. Morokuma, V. G. Zakrzewski, G. A. Voth, P. Salvador, J. J. Dannenberg, S. Dapprich, A. D. Daniels, Ö. Farkas, J. B. Foresman, J. V. Ortiz, J. Cioslowski, and D. J. Fox, GaUsSIAN 09, Gaussian, Inc., Wallingford, CT, USA, 2009.

${ }^{23}$ W. Holzer, A. Penzkofer, M. Fuhrmann, and P. Hegemann, Photochem. Photobiol. 75, 479 (2002).

${ }^{24}$ G. P. Laroff, R. W. Fessenden, and R. H. Schuler, J. Am. Chem. Soc. 94, 9062 (1972).

${ }^{25}$ S. Schreiner, U. Steiner, and H. E. A. Kramer, Photochem. Photobiol. 21, 81 (1975).

${ }^{26}$ P. F. Heelis, B. J. Parsons, G. O. Phillips, and J. F. Mckellar, Photochem. Photobiol. 28, 169 (1978).

${ }^{27}$ P. F. Heelis, B. J. Parsons, G. O. Phillips, and J. F. Mckellar, Photochem. Photobiol. 33, 7 (1981).

${ }^{28}$ A. Weller, F. Nolting, and H. Staerk, Chem. Phys. Lett. 96, 24 (1983).

${ }^{29}$ E. J. Land and A. J. Swallow, Biochemistry 8, 2117 (1969).

${ }^{30}$ A. I. Shushin, Chem. Phys. Lett. 181, 274 (1991).

${ }^{31}$ A. I. Shushin, J. B. Pedersen, and L. I. Lolle, Chem. Phys. 188, 1 (1994).
${ }^{32}$ T. Suzuki, T. Miura, K. Maeda, and T. Arai, J. Phys. Chem. A 109, 9911 (2005).

${ }^{33}$ N. N. Lukzen, J. B. Pedersen, and A. I. Burshtein, J. Phys. Chem. A 109, 11914 (2005).

${ }^{34}$ A. I. Burshtein, Adv. Chem. Phys. 114, 419 (2000).

${ }^{35}$ A. Rosspeintner, D. R. Kattnig, G. Angulo, S. Landgraf, and G. Grampp, Chem. - Eur. J. 14, 6213 (2008).

${ }^{36}$ P. A. Purtov and A. B. Doktorov, Chem. Phys. 178, 47 (1993).

${ }^{37}$ P. A. Purtov, A. B. Doktorov, and A. V. Popov, Chem. Phys. 182, 149 (1994).

${ }^{38}$ A. B. Doktorov and J. B. Pedersen, J. Chem. Phys. 108, 6868 (1998).

${ }^{39}$ A. A. Neufeld, A. B. Doktorov, and J. B. Pedersen, J. Chem. Phys. 115, 3219 (2001).

${ }^{40}$ K. L. Ivanov, N. N. Lukzen, H. M. Vieth, S. Grosse, A. V. Yurkovskaya, and R. Z. Sagdeev, Mol. Phys. 100, 1197 (2002).

${ }^{41}$ A. B. Doktorov and J. B. Pedersen, Chem. Phys. 322, 433 (2006).

${ }^{42}$ A. B. Doktorov, M. J. Hansen, and J. B. Pedersen, Chem. Phys. 328, 333 (2006).

${ }^{43}$ K. L. Ivanov, V. M. Sadovsky, and N. N. Lukzen, J. Chem. Phys. 143, 084110 (2015).

${ }^{44}$ R. Haberkorn, Mol. Phys. 32, 1491 (1976).

${ }^{45}$ R. Bittl, K. Schulten, and N. J. Turro, J. Chem. Phys. 93, 8260 (1990).

${ }^{46}$ Y. Kobori, S. Sekiguchi, K. Akiyama, and S. Tero-Kubota, J. Phys. Chem. A 103, 5416 (1999).

${ }^{47}$ D. R. Kattnig, A. Rosspeintner, and G. Grampp, Phys. Chem. Chem. Phys. 13, 3446 (2011).

${ }^{48}$ F. W. Byron and R. W. Fuller, The Mathematics of Classical and Quantum Physics (Dover Publications, New York, 1992).

${ }^{49}$ A. A. Zharikov and N. V. Shokhirev, Chem. Phys. Lett. 186, 253 (1991).

${ }^{50}$ D. Njus and P. M. Kelley, FEBS Lett. 284, 147 (1991).

${ }^{51}$ A. B. Doktorov and J. B. Pedersen, Chem. Phys. Lett. 423, 208 (2006).

${ }^{52}$ J. Mueller, e-print arXiv:1404.4261(2014).

${ }^{53}$ Surrogate models are used to find (near) optimal solutions of computationally expensive problems with a very limited number of function evaluations (Ref. 43).

${ }^{54}$ D. H. Macartney and N. Sutin, Inorg. Chim. Acta 74, 221 (1983).

${ }^{55}$ R. W. Eveson, C. R. Timmel, B. Brocklehurst, P. J. Hore, and K. A. McLauchlan, Int. J. Radiat. Biol. 76, 1509 (2000).

${ }^{56}$ M. Murakami, K. Maeda, and T. Arai, J. Phys. Chem. A 109, 5793 (2005).

${ }^{57}$ C. A. Dodson, C. J. Wedge, M. Murakami, K. Maeda, M. I. Wallace, and P. J. Hore, Chem. Commun. 51, 8023 (2015). 